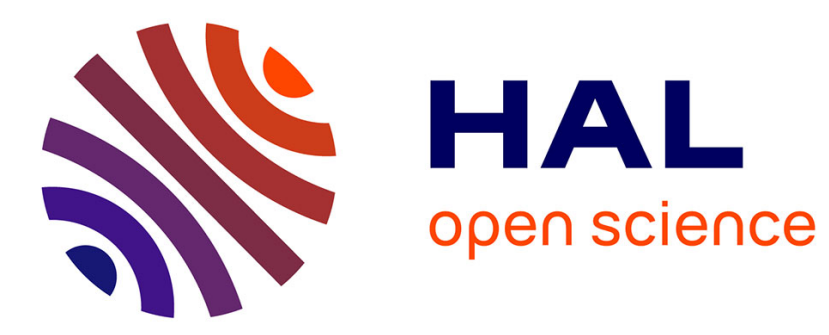

\title{
Foucault pendulum-like problems: A tensorial approach
}

Daniel Condurache, Vladimir Martinusi

\section{To cite this version:}

Daniel Condurache, Vladimir Martinusi. Foucault pendulum-like problems: A tensorial approach. International Journal of Non-Linear Mechanics, 2008, 43 (8), pp.743. 10.1016/j.ijnonlinmec.2008.03.009 . hal-00501784

\section{HAL Id: hal-00501784 \\ https://hal.science/hal-00501784}

Submitted on 12 Jul 2010

HAL is a multi-disciplinary open access archive for the deposit and dissemination of scientific research documents, whether they are published or not. The documents may come from teaching and research institutions in France or abroad, or from public or private research centers.
L'archive ouverte pluridisciplinaire HAL, est destinée au dépôt et à la diffusion de documents scientifiques de niveau recherche, publiés ou non, émanant des établissements d'enseignement et de recherche français ou étrangers, des laboratoires publics ou privés. 


\section{Author's Accepted Manuscript}

Foucault pendulum-like problems: A tensorial approach

Daniel Condurache, Vladimir Martinusi

PII:

S0020-7462(08)00065-6

DOI:

doi:10.1016/j.ijnonlinmec.2008.03.009

Reference:

NLM 1467

To appear in: International Journal of NonLinear Mechanics

Received date: 18 April 2006

Revised date: 24 January 2008

Accepted date: 31 March 2008
-

NON-LINEAR

MECHANICS

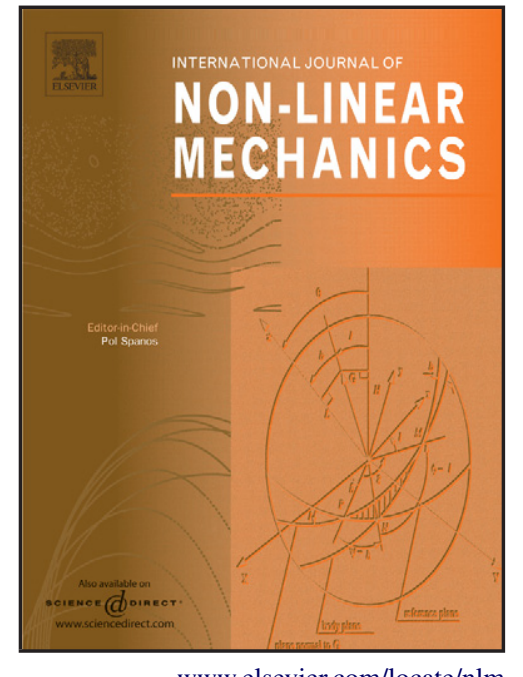

www.elsevier.com/locate/nlm

Cite this article as: Daniel Condurache and Vladimir Martinusi, Foucault pendulum-like problems: A tensorial approach, International Journal of Non-Linear Mechanics (2008), doi:10.1016/j.ijnonlinmec.2008.03.009

This is a PDF file of an unedited manuscript that has been accepted for publication. As a service to our customers we are providing this early version of the manuscript. The manuscript will undergo copyediting, typesetting, and review of the resulting galley proof before it is published in its final citable form. Please note that during the production process errors may be discovered which could affect the content, and all legal disclaimers that apply to the journal pertain. 


\title{
Foucault Pendulum-Like Problems: A Tensorial Approach
}

\author{
Daniel Condurache ${ }^{1}$ and Vladimir Martinusi ${ }^{2}$ \\ Technical University "Gheorghe Asachi" Iasi, Romania \\ Department of Theoretical Mechanics \\ E-mails: ${ }^{1}$ daniel.condurache@gmail.com, ${ }^{2}$ vladmartinus@gmail.com
}

\begin{abstract}
The paper offers a comprehensive study of the motion in a central force field with respect to a rotating noninertial reference frame. It is called Foucault Pendulum-like motion and it is a generalization of a classic Theoretical Mechanics problem. A closed form vectorial solution to this famous problem is presented. The vectorial time-explicit solution for the classic Foucault Pendulum problem is obtained as a particular case of the considerations made in the present aproach. Inedite conservation laws for the Foucault Pendulum-like motion are deduced by using simple differential and vectorial computations. They help to visualize the shape of the trajectories. Exact vectorial expressions for the law of motion and the velocity are also offered. The case of the driven Foucault Pendulum is also analyzed, and a closed form solution is deduced based on the general considerations. In the end, an inedite tensorial prime integral for the Foucault Pendulum problem is offered. It helps to reveal in a concise form, within a single entity, all the scalar and vectorial conservation laws for the Foucault Pendulum motion.

Two important engineering applications to this approach are presented: the motion of a satellite with respect to a rotating reference frame and the Keplerian relative orbital motion. The latter has a great importance in modeling the problems concerning satellite formation flying, satellite constellations and space terminal rendezvous. The classic problem of the harmonic oscillator in an electromagnetic field is also solved by using the instruments presented in this paper.

Keywords: Foucault Pendulum, orthogonal tensors, rotating reference frame, central force field, vectorial closed form solution, relative satellite motion.
\end{abstract}

\section{Introduction}

Vous êtes invités à venir voir tourner la Terre... are some of the words printed on a huge banner hanging outside the Panthéon in Paris.

In 1851, February 3, Jean Bernard Léon Foucault (1819 - 1868) used the same words to invite scientists of the day to come and witness his revolutionary experiment which would proof that Earth does indeed spin on its axis. The event, held at the Paris Observatory, had such an impact that Prince Louis Napoleon Bonaparte, the future Napoleon III, asked him to give a public demonstration a month later on 26 March.

The Foucault Pendulum was invented by accident in 1848, when French scientist Léon Foucault noticed that a small pendulum set oscillating in his rotating drill press completely ignores the rotation and keeps the oscillation plane fixed. He then constructed a two meters-long pendulum with a five kilogram ball in his workshop in the cellar. Before the amplitude of the swing completely died away, Foucault noticed that the weight on the end of the pendulum appeared to rotate clockwise. Now that he was convinced of the principle, he built a second pendulum with an 11-meters wire in Paris Observatory, and it too rotated clockwise. It proved that the pendulum completely ignores the local effects and its balancing plane lines itself with the Universe (see [1], [2]).

The present paper offers a complete study of the general framework of the Foucault Pendulum and introduces an instrument to solve this problem in a much more general situation. The approach determines a general method to deal with the motion in a central force field with respect to a rotating non-inertial reference frame. This type of motion will prove to be similar to the Foucault Pendulum. The problem is comprehensively studied and solved in the end, with closed form solutions in each case. In the particular case of the classic Foucault Pendulum, a time explicit solution is offered.

The type of motion that will be named Foucault Pendulum-like motion is described by the non-linear initial value 
problem:

$$
\left\{\begin{array}{l}
\ddot{\mathbf{r}}+2 \boldsymbol{\omega} \times \dot{\mathbf{r}}+\boldsymbol{\omega} \times(\boldsymbol{\omega} \times \mathbf{r})+\dot{\boldsymbol{\omega}} \times \mathbf{r}+f(r) \frac{\mathbf{r}}{r}=\mathbf{0}, \\
\mathbf{r}\left(t_{0}\right)=\mathbf{r}_{0}, \dot{\mathbf{r}}\left(t_{0}\right)=\mathbf{v}_{0},
\end{array}\right.
$$

where $f: \mathbb{R}_{+} \rightarrow \mathbb{R}$ is a continuous real valued map, $r$ denotes the magnitude of vector $\mathbf{r}$ and $\boldsymbol{\omega}$ a differentiable vectorial valued map ( $\mathbb{R}$ denotes the set of real numbers). Eq (1.1) models the motion with respect to a rotating reference frame with instantaneous angular velocity $\boldsymbol{\omega}$ under the influence of a central force field. Eq (1.1) also describes the motion of an Earth satellite with respect to a rotating reference frame with the origin in Earth center that rotates together with Earth. The relative orbital motion is modeled by an equation that is similar to eq (1.1) (see [3] [26]). This model is fundamental in spacecraft relative dynamics, an important problem in Celestial Mechanics and Astrodynamics, and the results have direct applications in orbital rendezvous (see [8,9]), satellite formation flying (see [37]-[39]) and satellite constellations (see [54]). Recently, it was proven by the authors of the present paper that the Keplerian relative orbital motion is similar to a Foucault Pendulum-like motion (see [21] - [26]). The relative orbital motion in a central force field is also a Foucault Pendulum-like motion, and it was studied in [25].

The type of motion that will be named Foucault Pendulum motion is described by the linear initial value problem:

$$
\left\{\begin{array}{l}
\ddot{\mathbf{r}}+2 \boldsymbol{\omega} \times \dot{\mathbf{r}}+\boldsymbol{\omega} \times(\boldsymbol{\omega} \times \mathbf{r})+\dot{\boldsymbol{\omega}} \times \mathbf{r}+\omega_{*}^{2} \mathbf{r}=\mathbf{0}, \omega_{*}>0 \\
\mathbf{r}\left(t_{0}\right)=\mathbf{r}_{0}, \dot{\mathbf{r}}\left(t_{0}\right)=\mathbf{v}_{0}
\end{array}\right.
$$

when the function $f$ from eq (1.1) has the particular expression $f(r)=\omega_{*}^{2} r$, with $\omega_{*}>0$ a constant real number. Here $\mathbf{r}$ represents the position vector, $\boldsymbol{\omega}$ is the angular velocity of the reference frame (an arbitrary differential vectorial map) and $\omega_{*}$ is the pulsation of the pendulum (it depends on its length and the gravitational acceleration at the experiment place). The initial value problem (1.2) describes a spatial harmonic oscillator related to an arbitrary rotating reference frame.

In Theoretical Mechanics textbooks (see for example [28]-[32]), the Foucault Pendulum problem (1.2) is reduced to the initial value problem:

$$
\left\{\begin{array}{l}
\ddot{\mathbf{r}}+2 \boldsymbol{\omega} \times \dot{\mathbf{r}}+\omega_{*}^{2} \mathbf{r}=\mathbf{0}, \omega_{*}>0 \\
\mathbf{r}\left(t_{0}\right)=\mathbf{r}_{0}, \dot{\mathbf{r}}\left(t_{0}\right)=\mathbf{v}_{0}
\end{array}\right.
$$

where vector $\boldsymbol{\omega}$ is considered to be constant, $\boldsymbol{\omega}=\boldsymbol{\omega}_{\text {Earth }} \sin \psi$, where $\psi$ is the latitude of the place where the Pendulum is situated and $\boldsymbol{\omega}_{\text {Earth }}$ represents the Earth angular velocity. Only the Coriolis force is taken into account in eq (1.3), the term $\boldsymbol{\omega} \times(\boldsymbol{\omega} \times \mathbf{r})$ being neglected. A vectorial closed form explicit solution is not presented by any work. Eq (1.3) also models the motion of a harmonic oscillator in a magnetic field. Traditionally, the solution to the initial value problem (1.3) is given only in the planar case, using polar coordinates (see [28]) or Cartesian coordinates (see [30]). V.I. Arnold gives an approximate solution to the initial value problem (1.3) using the isomorphism between plane vectors and complex numbers (see [29]). Eq (1.3) will be named Reduced Foucault Pendulum problem within this paper.

By using an adequate tensor instrument, a representation theorem for the Foucault Pendulum-like motion problem (1.1) is given in this paper. With the help of this result, two conservation laws of motion of eq (1.1) are deduced: one is analogous to the specific angular momentum conservation law and the other is a generalized specific energy conservation law. They allow the qualitative study of the motion described by eq (1.1) as well as to visualize the trajectories.

The method used in this paper consists in the reduction of the study of the motion described by the initial value problem (1.1) to the classic inertial problem of a motion in a central force field. This fact is accomplished through the use of a tensor coordinate-free method. The results that are deduced are presented in a closed vectorial form and they do not depend on a particular coordinate system that may be chosen (Cartesian, cylindrical, spherical) in the rotating reference frame.

By particularizing the method described above for the linear initial value problems (1.2) and (1.3), exact solutions in a vectorial closed form are offered to these equations. When the vectorial map $\boldsymbol{\omega}$ has a fixed direction, the solutions to eqs (1.2) and (1.3) become time-explicit.

This paper is structured as follows. Section 2 introduces the main instrument used: orthogonal and skewsymmetric tensors.

Section 3 offers a complete study of the Foucault Pendulum-like motions. Section 3.1 gives a representation theorem for the motion in a central force field with respect to a rotating reference frame with arbitrary instantaneous angular velocity. The motion is described by the initial value problem (1.1). 
This result shows that the Foucault Pendulum-like motion may be decomposed into:

(i) a planar (or rectilinear) and possibly periodic motion;

(ii) a precession with angular velocity $-\boldsymbol{\omega}$ of the plane (or of the straight line) around a fixed point.

This type of motion is characteristic to any motion in a central force field with respect to an arbitrary rotating reference frame.

The conservation laws of the Foucault Pendulum-like problem are presented in Section 3.2. The trajectories corresponding to the solution to eq (1.1) are presented in Section 3.3. Exact expressions for the law of motion and for the velocity are given in Section 3.4. Furthermore, some engineering applications are presented. The Foucault Pendulum-like motion and the orbital motion of an Earth satellite are linked in Section 3.5. The tensor method previously introduced allows to present a closed form exact solution to the relative Keplerian orbital motion problem, which is done in Section 3.6.

Section 4 offers the vectorial closed form solution to the Foucault Pendulum problem modeled by eq (1.2) in the most general case, when $\boldsymbol{\omega}$ is arbitrary. This solution is then particularized to explicit expressions for $\boldsymbol{\omega}$ having constant direction and $\boldsymbol{\omega}$ being constant. A generalization of the Chevillet Theorem is also offered in this Section. All possible cases are studied and suggestive visualizations of the motion are offered in Section 4.1. Section 5 gives an exact vectorial solution to the reduced Foucault Pendulum problem (1.3). The problem is solved in Theoretical Mechanics textbooks only in the planar case.

Section 6 gives the vectorial closed form solution to the driven Foucault Pendulum. Section 6.1 presents the closed form solution to the initial value problem (which is closer to the real model of the motion):

$$
\left\{\begin{array}{l}
\ddot{\mathbf{r}}+2 \boldsymbol{\omega} \times \dot{\mathbf{r}}+\boldsymbol{\omega} \times(\boldsymbol{\omega} \times \mathbf{r})+\dot{\boldsymbol{\omega}} \times \mathbf{r}+\omega_{*}^{2} \mathbf{r}=\mathbf{f}(t) \\
\mathbf{r}\left(t_{0}\right)=\mathbf{r}_{0} ; \dot{\mathbf{r}}\left(t_{0}\right)=\mathbf{v}_{0}
\end{array}\right.
$$

and Section 6.2 offers the closed form solution to the driven reduced Foucault Pendulum problem:

$$
\left\{\begin{array}{l}
\ddot{\mathbf{r}}+2 \boldsymbol{\omega} \times \dot{\mathbf{r}}+\omega_{*}^{2} \mathbf{r}=\mathbf{f}(t), \omega_{*}>0 \\
\mathbf{r}\left(t_{0}\right)=\mathbf{r}_{0}, \dot{\mathbf{r}}\left(t_{0}\right)=\mathbf{v}_{0}
\end{array}\right.
$$

where $\mathbf{f}: \mathbb{R} \rightarrow \mathbf{V}_{3}$ is a continuous vectorial map ( $\mathbf{V}_{3}$ the three-dimensional vectorial space). Eq (1.5) also models the motion of an electrical charged particle in an electromagnetic field.

Section 7 gives a tensor conservation law of the Foucault Pendulum problem that contains all its scalar and vectorial conservation laws. To the knowledge of the authors, this result is completely new. It was obtained by using the tensor instrument introduced in Section 2.

\section{Tensorial Considerations}

This section introduces a tensor method that may be used in the study of the general motion with respect to a noninertial rotating reference frame (see also [21], [24], [50]-[53]). Orthogonal and skew-symmetric tensor maps are the main elements of this approach.

We will denote by $\mathbf{S O}_{3}$ the special orthogonal group of second order tensors (rotation tensors), by $\mathbf{S O}_{3}^{\mathbb{R}}$ the set of the maps defined on $\mathbb{R}$ with values in $\mathbf{S O}_{3}$, by $\mathbf{s o}_{3}$ the group of skew-symmetric second order tensors and by $\mathbf{s o} \mathbf{R}_{3}^{\mathbb{R}}$ the set of maps defined on $\mathbb{R}$ with values in so s $_{3}$ (see also [29], [45], [46], [47]).

$$
\begin{aligned}
\mathbf{S O}_{3} & =\left\{\mathbf{R} \in L\left(\mathbf{V}_{3}\right) \mid \mathbf{R}^{T} \mathbf{R}=\mathbf{I}_{3}, \operatorname{det} \mathbf{R}=1\right\} ; \\
\mathbf{s o}_{3} & =\left\{\widetilde{\boldsymbol{\omega}} \in L\left(\mathbf{V}_{3}\right) \mid \widetilde{\boldsymbol{\omega}}^{T}=-\widetilde{\boldsymbol{\omega}}\right\} ; \\
\mathbf{S O}_{3}^{\mathbb{R}} & =\left\{\mathbf{R}: \mathbb{R} \rightarrow \mathbf{S O}_{3}\right\} ; \\
\mathbf{s o}_{3}^{\mathbb{R}} & =\left\{\widetilde{\boldsymbol{\omega}}: \mathbb{R} \rightarrow \mathbf{s o}_{3}\right\},
\end{aligned}
$$

where $L\left(\mathbf{V}_{3}\right)$ denotes the set of second order tensors, $\mathbf{I}_{3} \in L\left(\mathbf{V}_{3}\right)$ is the unit tensor, $\mathbf{R}^{T}$ denotes the transpose of tensor $\mathbf{R}$ and $\mathbb{R}$ denotes the set of real numbers.

There exists a bijective correspondence between $\mathbf{s o}_{3}$ and $\mathbf{V}_{3}$ : for a vector $\boldsymbol{\omega}=\left[\begin{array}{lll}\omega_{1} & \omega_{2} & \omega_{3}\end{array}\right]^{T} \in \mathbf{V}_{3}$, there exists a tensor in $\mathbf{s o}_{3}$

$$
\widetilde{\boldsymbol{\omega}}=\left[\begin{array}{ccc}
0 & -\omega_{3} & \omega_{2} \\
\omega_{3} & 0 & -\omega_{1} \\
-\omega_{2} & \omega_{1} & 0
\end{array}\right]
$$


such that for any vector $\mathbf{x} \in \mathbf{V}_{3}$, it holds true: $\widetilde{\boldsymbol{\omega}} \mathbf{x}=\boldsymbol{\omega} \times \mathbf{x}$.

The next theorem refers to the famous Darboux problem (see $[42,43,44])$ : finding the rotation tensor map when knowing the instantaneous angular velocity. This is a classic problem in rigid body kinematics.

Theorem 2.0.1 (Darboux). The initial value problem:

$$
\left\{\begin{array}{l}
\dot{\mathbf{Q}}=\mathbf{Q} \widetilde{\boldsymbol{\omega}} \\
\mathbf{Q}\left(t_{0}\right)=\mathbf{I}_{3}
\end{array}\right.
$$

with $t_{0} \geq 0$ has a unique solution $\mathbf{Q} \in \mathbf{S O}_{3}^{\mathbb{R}}$ for any continuous map $\widetilde{\boldsymbol{\omega}} \in \mathbf{\mathbf { s o } _ { 3 } ^ { \mathbb { R } }}$.

Proof. Denote $\mathbf{Q}^{T}$ the transpose of tensor $\mathbf{Q}$. Computing:

$$
\frac{d}{d t}\left(\mathbf{Q} \mathbf{Q}^{T}\right)=\dot{\mathbf{Q}} \mathbf{Q}^{T}+\mathbf{Q} \dot{\mathbf{Q}}^{T}=\mathbf{Q} \widetilde{\omega} \mathbf{Q}^{T}-\mathbf{Q} \widetilde{\omega} \mathbf{Q}^{T}=\mathbf{0}_{3},
$$

it follows that:

$$
\mathbf{Q Q}^{T}=\mathbf{Q Q}^{T}\left(t_{0}\right)=\mathbf{I}_{3} .
$$

Since $\mathbf{Q}=\mathbf{Q}(t)$ is a continuous map, $t \geq t_{0}$, it follows that $\operatorname{det}(\mathbf{Q})$ is a continuous map too. From eq (2.4) it results $\operatorname{det}(\mathbf{Q}) \in\{-1,1\}$. Since $\operatorname{det}\left(\mathbf{Q}\left(t_{0}\right)\right)=\operatorname{det} \mathbf{I}_{3}=1$, it follows that:

$$
\left\{\begin{array}{l}
\mathbf{Q Q}^{T}=\mathbf{I}_{3} \\
\operatorname{det}(\mathbf{Q})=1
\end{array}\right.
$$

therefore $\mathbf{Q} \in \mathbf{S O}_{3}^{\mathbb{R}}$ is a proper orthogonal tensor map.

We denote by $\mathbf{V}_{3}^{\mathbb{R}}$ the set of the maps defined on $\mathbb{R}$ with values in $\mathbf{V}_{3}$. The unique solution to the initial value problem (2.2) will be denoted $\mathbf{F}_{\boldsymbol{\omega}}$. The tensor map $\mathbf{F}_{\boldsymbol{\omega}}$ satisfies:

(i) $\mathbf{F}_{\boldsymbol{\omega}}$ is invertible and $\mathbf{F}_{\boldsymbol{\omega}}^{-1}=\mathbf{F}_{\boldsymbol{\omega}}^{T}$.

(ii) $\mathbf{F}_{\boldsymbol{\omega}} \mathbf{u} \cdot \mathbf{F}_{\boldsymbol{\omega}} \mathbf{v}=\mathbf{u} \cdot \mathbf{v},(\forall) \mathbf{u}, \mathbf{v} \in \mathbf{V}_{3}^{\mathbb{R}}$

(iii) $\left|\mathbf{F}_{\boldsymbol{\omega}} \mathbf{u}\right|=|\mathbf{u}|,(\forall) \mathbf{u} \in \mathbf{V}_{3}^{\mathbb{R}}$

(iv) $\mathbf{F}_{\boldsymbol{\omega}}(\mathbf{u} \times \mathbf{v})=\mathbf{F}_{\boldsymbol{\omega}} \mathbf{u} \times \mathbf{F}_{\boldsymbol{\omega}} \mathbf{v},(\forall) \mathbf{u}, \mathbf{v} \in \mathbf{V}_{3}^{\mathbb{R}}$

(v) $\frac{d}{d t} \mathbf{F}_{\boldsymbol{\omega}} \mathbf{u}=\mathbf{F}_{\boldsymbol{\omega}}(\dot{\mathbf{u}}+\boldsymbol{\omega} \times \mathbf{u}),(\forall) \mathbf{u} \in \mathbf{V}_{3}^{\mathbb{R}}$, differentiable;

$(v i) \frac{d^{2}}{d t^{2}} \mathbf{F}_{\boldsymbol{\omega}} \mathbf{u}=\mathbf{F}_{\boldsymbol{\omega}}(\ddot{\mathbf{u}}+2 \boldsymbol{\omega} \times \dot{\mathbf{u}}+\boldsymbol{\omega} \times(\boldsymbol{\omega} \times \mathbf{u})+\dot{\boldsymbol{\omega}} \times \mathbf{u}),(\forall) \mathbf{u} \in \mathbf{V}_{3}^{\mathbb{R}}$, differentiable.

A complete proof of properties $(i)-(i v)$ may be found in [29]. Properties $(v)-(v i)$ may be directly deduced by doing some computations based on Theorem 2.0.1.

The inverse of $\mathbf{F}_{\boldsymbol{\omega}}$ is denoted $\mathbf{R}_{-\boldsymbol{\omega}}$ :

$$
\left(\mathbf{F}_{\boldsymbol{\omega}}\right)^{-1} \stackrel{\text { not }}{=} \mathbf{R}_{-\boldsymbol{\omega}}
$$

The tensor map $\mathbf{R}_{-\boldsymbol{\omega}}$ describes the rotation with angular velocity $\boldsymbol{-} \boldsymbol{\omega}$. It plays a fundamental role in this paper.

As $\mathbf{F}_{\boldsymbol{\omega}}$ is the solution to the initial value problem (2.2), then $\mathbf{R}_{-\boldsymbol{\omega}}$ satisfies:

$$
\left\{\begin{array}{l}
\dot{\mathbf{R}}_{-\boldsymbol{\omega}}+\widetilde{\boldsymbol{\omega}} \mathbf{R}_{-\boldsymbol{\omega}}=\mathbf{0} \\
\mathbf{R}_{-\boldsymbol{\omega}}\left(t_{0}\right)=\mathbf{I}_{3}
\end{array}\right.
$$

We denote by $\boldsymbol{\omega}$ the vectorial map associated to the skew-symmetric tensor map $\widetilde{\boldsymbol{\omega}}$. Remark that when $\boldsymbol{\omega}$ has constant direction, $\boldsymbol{\omega}=\omega \mathbf{u}$, with $\mathbf{u}$ its constant unit vector and $\omega: \mathbb{R} \rightarrow \mathbb{R}$ a scalar function, since $\widetilde{\boldsymbol{\omega}}\left(t_{1}\right) \widetilde{\boldsymbol{\omega}}\left(t_{2}\right)=$ $\widetilde{\boldsymbol{\omega}}\left(t_{2}\right) \widetilde{\boldsymbol{\omega}}\left(t_{1}\right),(\forall) t_{1,2} \in \mathbb{R}$, then $\mathbf{R}_{-\boldsymbol{\omega}(t)}=\exp \left(-\int_{t_{0}}^{t} \widetilde{\boldsymbol{\omega}}(\xi) d \xi\right)$ (see [21], [26], [48]) and it has the explicit expression:

$$
\mathbf{R}_{-\boldsymbol{\omega}(t)}=\mathbf{I}_{3}-\frac{\sin \varphi(t)}{\omega} \widetilde{\boldsymbol{\omega}}+\frac{1-\cos \varphi(t)}{\omega^{2}} \widetilde{\boldsymbol{\omega}}^{2},
$$

where $\varphi(t)=\int_{t_{0}}^{t} \omega(\xi) d \xi$. If $\boldsymbol{\omega}$ is constant then $\mathbf{R}_{-\boldsymbol{\omega}(t)}$ has the explicit form:

$$
\mathbf{R}_{-\boldsymbol{\omega}(t)}=\exp \left[-\left(t-t_{0}\right) \widetilde{\boldsymbol{\omega}}\right]=\mathbf{I}_{3}-\frac{\sin \left[\omega\left(t-t_{0}\right)\right]}{\omega} \widetilde{\boldsymbol{\omega}}+\frac{1-\cos \left[\omega\left(t-t_{0}\right)\right]}{\omega^{2}} \widetilde{\boldsymbol{\omega}}^{2} .
$$


If vector $\boldsymbol{\omega}$ has a regular precession, $\boldsymbol{\omega}=\boldsymbol{\omega}_{1}+\mathbf{R}_{-\boldsymbol{\omega}_{1}} \boldsymbol{\omega}_{2}$, with $\boldsymbol{\omega}_{1,2} \in \mathbf{V}_{3}$ constant vectors, then $\mathbf{R}_{-\boldsymbol{\omega}(t)}$ has the explicit form (see $[21])$ :

$$
\mathbf{R}_{-\boldsymbol{\omega}(t)}=\exp \left[-\left(t-t_{0}\right) \widetilde{\boldsymbol{\omega}}_{1}\right] \cdot \exp \left[-\left(t-t_{0}\right) \widetilde{\boldsymbol{\omega}}_{2}\right] .
$$

More detailed approaches may be found in [33] - [36].

\section{The Foucault Pendulum-Like Problem: A Complete Study}

In this section a representation theorem of the general motion in a rotating reference frame under the influence of a central positional force is given. Conservation laws of the Foucault Pendulum-like motion are deduced. They allow a qualitative analysis of the motion and the visualization of the trajectory. Exact vectorial expressions for the law of motion and for the velocity are then deduced.

\subsection{From Non-Inertial to Inertial Motion: A Smooth Transfer}

A particle is considered to move under the influence of a central positional force field, i.e. the force that has the vectorial expression:

$$
\mathbf{F}=-m f(r) \frac{\mathbf{r}}{r}
$$

where $m$ is the mass of the particle and $f: \mathbb{R}_{+} \rightarrow \mathbb{R}$ is a continuous map that depends on the magnitude of the position vector. The initial value problem that describes the motion of a particle with respect to a rotating reference frame under the influence of a force described by eq (3.1) is eq (1.1). A representation theorem related to this type of motion may be obtained. This result was first given in another context in $[25,50]$. It states that the solution to the initial value problem:

$$
\left\{\begin{array}{l}
\ddot{\mathbf{r}}+2 \boldsymbol{\omega} \times \dot{\mathbf{r}}+\boldsymbol{\omega} \times(\boldsymbol{\omega} \times \mathbf{r})+\dot{\boldsymbol{\omega}} \times \mathbf{r}+f(r) \frac{\mathbf{r}}{r}=\mathbf{0}, \\
\mathbf{r}\left(t_{0}\right)=\mathbf{r}_{0} ; \dot{\mathbf{r}}\left(t_{0}\right)=\mathbf{v}_{0},
\end{array}\right.
$$

is obtained by applying the tensor map $\mathbf{R}_{-\boldsymbol{\omega}}$ to the solution to the initial value problem:

$$
\left\{\begin{array}{l}
\ddot{\mathbf{r}}+f(r) \frac{\mathbf{r}}{r}=\mathbf{0}, \\
\mathbf{r}\left(t_{0}\right)=\mathbf{r}_{0} ; \dot{\mathbf{r}}\left(t_{0}\right)=\mathbf{v}_{0}+\boldsymbol{\omega}_{0} \times \mathbf{r}_{0},
\end{array}\right.
$$

where $\boldsymbol{\omega}$ represents the instantaneous angular velocity of the reference frame and $\boldsymbol{\omega}_{0}=\boldsymbol{\omega}\left(t_{0}\right)$.

Below we present a concise proof to this statement.

By applying the tensorial operator $\mathbf{F}_{\boldsymbol{\omega}}$ and taking into account its properties, the initial value problem (3.2) may be written as:

$$
\left\{\begin{array}{l}
\frac{d^{2}}{d t^{2}}\left(\mathbf{F}_{\boldsymbol{\omega}} \mathbf{r}\right)+f\left(\left|\mathbf{F}_{\boldsymbol{\omega}} \mathbf{r}\right|\right) \frac{\mathbf{F}_{\boldsymbol{\omega}} \mathbf{r}}{\left|\mathbf{F}_{\boldsymbol{\omega}} \mathbf{r}\right|}=\mathbf{0} \\
\left(\mathbf{F}_{\boldsymbol{\omega}} \mathbf{r}\right)\left(t_{0}\right)=\mathbf{r}_{0},\left[\frac{d}{d t}\left(\mathbf{F}_{\boldsymbol{\omega}} \mathbf{r}\right)\right]\left(t_{0}\right)=\mathbf{v}_{0}+\boldsymbol{\omega}_{0} \times \mathbf{r}_{0}
\end{array}\right.
$$

It results that if $\mathbf{r}$ is the solution to the initial value problem (3.2), then $\mathbf{F}_{\boldsymbol{\omega}} \mathbf{r}$ is the solution to the initial value problem (3.3). Since the tensorial map $\mathbf{F}_{\boldsymbol{\omega}}$ in invertible and its inverse is $\left(\mathbf{F}_{\boldsymbol{\omega}}\right)^{-1}=\mathbf{R}_{-\boldsymbol{\omega}}$, it results that if $\mathbf{r}$ is the solution to the initial value problem (3.3), then $\mathbf{R}_{-\omega} \mathbf{r}$ is the solution to the initial value problem (3.2).

Remark that the study of the non-linear initial value problem with variable coefficients (3.2) is reduced to the study of the motion in a central force field described by eq (3.3).

This method is purely symbolic and it offers the way to solve most non-inertial problems. By applying the tensor map $\mathbf{F}_{\boldsymbol{\omega}}$ to the initial value problem (3.2), the problem is transferred to an inertial reference frame. The solution to the new problem is determined with respect to this reference frame and then it is transferred back to the non-inertial 
frame by the inverse tensor operator that is $\mathbf{R}_{-\boldsymbol{\omega}}$. The inertial frame that appears here (its existence is postulated by the Inertia Principle) plays only a catalyst role in this symbolic method. Finally, the solution to the non-inertial initial value problem is expressed in the original non-inertial reference frame (see Figure 1). This procedure is similar to the Laplace and Fourier transforms in the case of the systems of linear differential equations.

From the mathematical point of view, the change of variable:

$$
\mathbf{r} \rightarrow \mathbf{F}_{\boldsymbol{\omega}} \mathbf{r}
$$

makes vanish all non-inertial terms which are present in the initial value problem that describes the motion. The transformation modifies both the differential equation and the initial conditions.

The initial condition $\dot{\mathbf{r}}\left(t_{0}\right)=\mathbf{v}_{0}+\boldsymbol{\omega}_{0} \times \mathbf{r}_{0}$ represents the inertial absolute velocity at $t=t_{0}$. After solving the

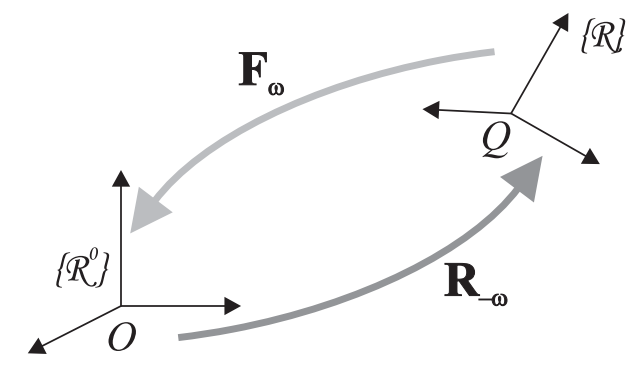

Figure 1: The transforms between a non-inertial reference frame and the inertial one.

"inertial" initial value problem, the return in the non-inertial frame is done by the inverse transform:

$$
\mathbf{r} \rightarrow \mathbf{R}_{-\omega} \mathbf{r}
$$

One may remark that the hodograph of the solution to the initial value problem (3.3) is a plane curve if $\mathbf{r}_{0} \times$ $\left(\mathbf{v}_{0}+\boldsymbol{\omega}_{0} \times \mathbf{r}_{0}\right) \neq \mathbf{0}$ or a line segment if $\mathbf{r}_{0} \times\left(\mathbf{v}_{0}+\boldsymbol{\omega}_{0} \times \mathbf{r}_{0}\right)=\mathbf{0}$. In fact, the motion described by the initial value problem (3.2) may be decomposed into:

( $i$ ) a planar (or rectilinear) and possibly periodic motion;

(ii) a precession with angular velocity $-\boldsymbol{\omega}$ of the plane (or of the straight line) around a fixed point.

\subsection{The Conservation Laws of the Foucault Pendulum-Like Motion}

The qualitative aspects of the motion may be studied by using the conservation laws of the initial value problem (3.2), which are presented in this section. These laws will be deduced by using the method introduced in Section 2.

We state that the conservation laws of the initial value problem (3.2) are:

$$
\mathbf{r} \times(\dot{\mathbf{r}}+\boldsymbol{\omega} \times \mathbf{r})=\mathbf{R}_{-\boldsymbol{\omega}} \boldsymbol{\Omega}_{0} \stackrel{\text { def }}{=} \boldsymbol{\Omega}
$$

where:

$$
\boldsymbol{\Omega}_{0}=\mathbf{r}_{0} \times\left(\mathbf{v}_{0}+\omega_{0} \times \mathbf{r}_{0}\right)
$$

(analogous to the specific angular momentum conservation);

$$
\frac{\dot{\mathbf{r}}^{2}}{2}+(\boldsymbol{\omega}, \mathbf{r}, \dot{\mathbf{r}})+\frac{1}{2}(\boldsymbol{\omega} \times \mathbf{r})^{2}+U(r) \stackrel{\text { def }}{=} h,
$$

where:

$$
h=\frac{\mathbf{v}_{0}^{2}}{2}+\left(\boldsymbol{\omega}, \mathbf{r}_{0}, \mathbf{v}_{0}\right)+\frac{1}{2}\left(\boldsymbol{\omega}_{0} \times \mathbf{r}_{0}\right)^{2}+U\left(r_{0}\right)
$$

(analogous to the specific energy conservation), with $U(r)=\int f(r) d r$ an antiderivative of map $f$. Here $(\boldsymbol{\omega}, \mathbf{r}, \dot{\mathbf{r}})$ denotes the triple scalar product of vectors $\boldsymbol{\omega}, \mathbf{r}, \dot{\mathbf{r}}:(\boldsymbol{\omega}, \mathbf{r}, \dot{\mathbf{r}}) \stackrel{\text { def }}{=} \boldsymbol{\omega} \cdot(\mathbf{r} \times \dot{\mathbf{r}})$. 
Below we present a proof to the validity of eqs (3.7) and.(3.8).

It is known [32] that a conservation law of the initial value problem (3.3) is:

$$
\mathbf{r} \times \dot{\mathbf{r}}=\mathbf{r}_{0} \times\left(\mathbf{v}_{0}+\boldsymbol{\omega}_{0} \times \mathbf{r}_{0}\right) \stackrel{\text { def }}{=} \boldsymbol{\Omega}_{0} .
$$

(the conservation law of the specific angular momentum). Since the solution to the initial value problem (3.2) is obtained by applying the tensor operator $\mathbf{F}_{\boldsymbol{\omega}}$ to the solution to the initial value problem (3.3), it follows that if $\mathbf{r}$ is solution to this initial value problem, then:

$$
\mathbf{F}_{\boldsymbol{\omega}} \mathbf{r} \times \frac{d}{d t}\left(\mathbf{F}_{\boldsymbol{\omega}} \mathbf{r}\right)=\boldsymbol{\Omega}_{0} .
$$

It follows that:

$$
\mathbf{F}_{\boldsymbol{\omega}}[\mathbf{r} \times(\dot{\mathbf{r}}+\boldsymbol{\omega} \times \mathbf{r})]=\boldsymbol{\Omega}_{0} \Rightarrow \mathbf{r} \times(\dot{\mathbf{r}}+\boldsymbol{\omega} \times \mathbf{r})=\mathbf{R}_{-\boldsymbol{\omega}} \boldsymbol{\Omega}_{0} .
$$

Eq (3.8) is deduced by starting from the conservation law [32]:

$$
\frac{1}{2} \dot{\mathbf{r}}^{2}+U(r)=\text { constant }
$$

of the initial value problem (3.3), where $U(r)=\int f(r) d r$ (the conservation law of the specific energy). Taking into account that the solution to eq (3.2) is obtained by applying the tensor operator $\mathbf{F}_{\boldsymbol{\omega}}$ to the solution to eq (3.3), it results that eq (3.12) may be written as:

$$
\frac{1}{2}\left[\frac{d}{d t}\left(\mathbf{F}_{\boldsymbol{\omega}} \mathbf{r}\right)\right]^{2}+U(r)=\text { constant }
$$

Taking into account the property $(v)$ of $\mathbf{F}_{\boldsymbol{\omega}}$, eq (3.13) transforms into:

$$
\frac{1}{2}(\dot{\mathbf{r}}+\boldsymbol{\omega} \times \mathbf{r})^{2}+U(r)=\text { constant } .
$$

After computations, it follows that eq (3.8) holds true. More detailed approaches may be found in [21, 50].

One may remark that vector $\boldsymbol{\Omega}$ is analogous to the specific angular momentum of the motion in a central force field. As it follows from eq (3.7), it is a vector that rotates with angular velocity $\boldsymbol{-} \boldsymbol{\omega}$. Due to this fact, only the magnitude of the vector function $\Omega$ remains constant.

If $\boldsymbol{\Omega}_{0} \neq \mathbf{0} \Leftrightarrow \boldsymbol{\Omega} \neq \mathbf{0}$, from eq (3.7) it follows that the hodograph of the vectorial map $\boldsymbol{\Omega}$ is a spherical curve (see Figure 2). When vector $\boldsymbol{\omega}$ has constant direction, $\boldsymbol{\Omega}$ has a precession with angular velocity $\boldsymbol{-} \boldsymbol{\omega}$. If vector $\boldsymbol{\omega}$ is constant then the precession is regular. If $\boldsymbol{\Omega}_{0}=\mathbf{0} \Leftrightarrow \boldsymbol{\Omega}=\mathbf{0}$, then the following conservation law of eq (3.2) holds:
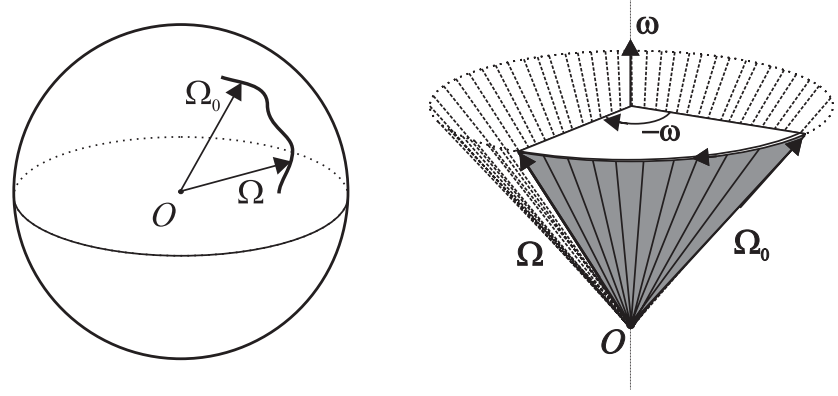

Figure 2: Left: when $\boldsymbol{\Omega}_{0} \neq \mathbf{0}$, the hodograph of map $\boldsymbol{\Omega}$ is a spherical curve. Right: if $\boldsymbol{\omega}$ has constant direction, vector $\boldsymbol{\Omega}$ "sweeps" the lateral surface of a right circular cone.

$$
\mathbf{r} \times(\dot{\mathbf{r}}+\boldsymbol{\omega} \times \mathbf{r})=\mathbf{0} .
$$

In this case, the hodograph of the solution to eq (3.3) is a line segment. 
Eq (3.8) represents the generalized energy conservation law. By denoting:

$$
\left\{\begin{array}{l}
E_{k i n}=\frac{m \dot{\mathbf{r}}^{2}}{2} \\
V(t, \mathbf{r}, \dot{\mathbf{r}})=m(\boldsymbol{\omega}, \mathbf{r}, \dot{\mathbf{r}})+\frac{m}{2}(\boldsymbol{\omega} \times \mathbf{r})^{2}+m U(r),
\end{array}\right.
$$

with $m$ the mass of the body under study, eq (3.8) may be rewritten as:

$$
E_{k i n}+V(t, \mathbf{r}, \dot{\mathbf{r}})=\text { constant }
$$

$E_{k i n}$ represents the kinetic energy of the body in the rotating reference frame and $V(t, \mathbf{r}, \dot{\mathbf{r}})$ the generalized potential energy with respect to the rotating reference frame. The following remarks are to be done:

1. When the angular velocity $\boldsymbol{\omega}$ has constant direction, taking into account that $\mathbf{R}_{-\boldsymbol{\omega}} \boldsymbol{\omega}=\boldsymbol{\omega}$, it results:

$$
(\boldsymbol{\omega}, \mathbf{r}, \dot{\mathbf{r}}+\boldsymbol{\omega} \times \mathbf{r})=\boldsymbol{\omega} \cdot \mathbf{R}_{-\boldsymbol{\omega}} \boldsymbol{\Omega}_{0}=\boldsymbol{\omega} \cdot \boldsymbol{\Omega}_{0}
$$

The conservation law (3.8) becomes:

$$
E_{k i n}+V(t, \mathbf{r})=\text { constant, where }\left\{\begin{array}{l}
V(t, \mathbf{r})=m\left[\boldsymbol{\Omega}_{0} \cdot \boldsymbol{\omega}-\frac{1}{2}(\boldsymbol{\omega} \times \mathbf{r})^{2}+U(r)\right], \\
\boldsymbol{\Omega}_{0}=\mathbf{r}_{0} \times\left(\mathbf{v}_{0}+\boldsymbol{\omega}_{0} \times \mathbf{r}_{0}\right) .
\end{array}\right.
$$

2. If the angular velocity $\boldsymbol{\omega}$ is constant:

$$
E_{k i n}+V(\mathbf{r})=\text { constant, where } V(\mathbf{r})=m\left[U(r)-\frac{(\boldsymbol{\omega} \times \mathbf{r})^{2}}{2}\right] .
$$

In this particular case, $V(\mathbf{r})$ represents the classic potential energy.

\subsection{The Trajectories in the Foucault Pendulum-Like Motion}

In the study of the trajectories of the Foucault Pendulum-like motion two distinct situations may occur:

1. When $\boldsymbol{\Omega}_{0} \neq \mathbf{0}$, the trajectory may be visualized as it follows: at $t=t_{0}$, a plane $\boldsymbol{\Pi}\left(t_{0}\right)$ is formed. It is uniquely determined by the initial conditions $\mathbf{r}_{0}, \mathbf{v}_{0}, \boldsymbol{\omega}_{0}$ and has the vectorial equation:

$$
\mathbf{r} \cdot \boldsymbol{\Omega}_{0}=0, O \in \boldsymbol{\Pi}\left(t_{0}\right) .
$$

This plane starts rotating with angular velocity $-\boldsymbol{\omega}$ around the fixed point $O$. At time $t$, the vectorial equation of plane $\Pi(t)$ is:

$$
\mathbf{r} \cdot \boldsymbol{\Omega}=0, O \in \Pi(t) .
$$

The motion of the particle with respect to plane $\boldsymbol{\Pi}(t)$ is described by the initial value problem (3.3).

2. If $\boldsymbol{\Omega}_{0}=\mathbf{0}$, a straight line $d\left(t_{0}\right)$ is considered to be instead of a plane. The vectorial equation of $d\left(t_{0}\right)$ is:

$$
\mathbf{r} \times \frac{\mathbf{r}_{0}}{r_{0}}=\mathbf{0}, O \in d\left(t_{0}\right) .
$$

This straight line begins to rotate with angular velocity $-\boldsymbol{\omega}$ around point $O$. At time $t$, the vectorial equation of $d(t)$ is:

$$
\mathbf{r} \times \mathbf{R}_{-\omega} \frac{\mathbf{r}_{0}}{r_{0}}=\mathbf{0}, O \in d(t) .
$$

The motion of the particle with respect to the straight line $d(t)$ is described by the initial value problem (3.3). 


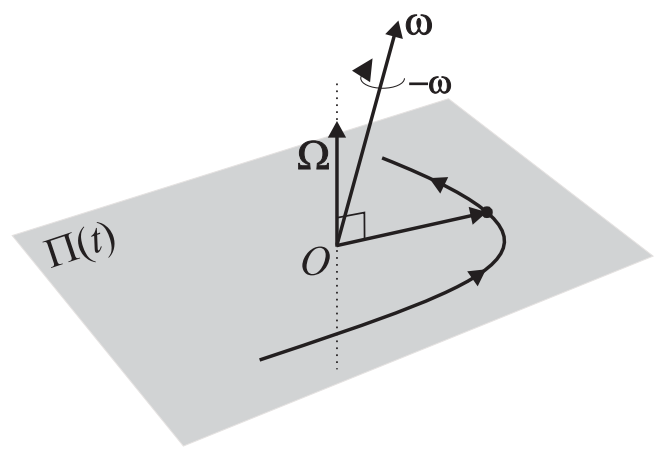

Figure 3: The generic trajectory of the Foucault Pendulum-like motion.

\subsubsection{Non-Zero Generalized Angular Momentum: $\Omega \neq 0$}

The motion may be visualized as it is described by eq (3.3) in a variable plane $\Pi(t)$ that rotates with angular velocity $-\boldsymbol{\omega}$ around a fixed point. Vector $\boldsymbol{\Omega}$ is normal to this plane at any moment of time, as it follows from eq (3.7) (see Figure 3$)$. The vectorial equation of plane $\boldsymbol{\Pi}(t)$ at $t \geq t_{0}$ is given in eq (3.22).

Generally, the trajectory is a space curve. It may be planar, depending on vector $\boldsymbol{\omega}$ and the initial conditions.

One may remark that if vector $\boldsymbol{\omega}$ has a fixed direction and $\boldsymbol{\omega} \times \boldsymbol{\Omega}_{0}=\mathbf{0}$, it follows that $\boldsymbol{\Omega}_{0}=\lambda \boldsymbol{\omega}$, where $\lambda: \mathbb{R} \rightarrow \mathbb{R}$ is a continuous map. In this particular case, it also holds true that $\mathbf{R}_{-\boldsymbol{\omega}} \boldsymbol{\omega}=\boldsymbol{\omega}$. It follows that

$$
\boldsymbol{\Omega}=\mathbf{R}_{-\boldsymbol{\omega}} \boldsymbol{\Omega}_{0}=\lambda \mathbf{R}_{-\boldsymbol{\omega}} \boldsymbol{\omega}=\lambda \boldsymbol{\omega}=\boldsymbol{\Omega}_{0},
$$

so vector $\boldsymbol{\Omega}$ is constant. Since it is normal to the plane $\boldsymbol{\Pi}(t)$, then the planar direction of $\boldsymbol{\Pi}(t)$ remains constant. The trajectory results to be a plane curve (see Figure 4).

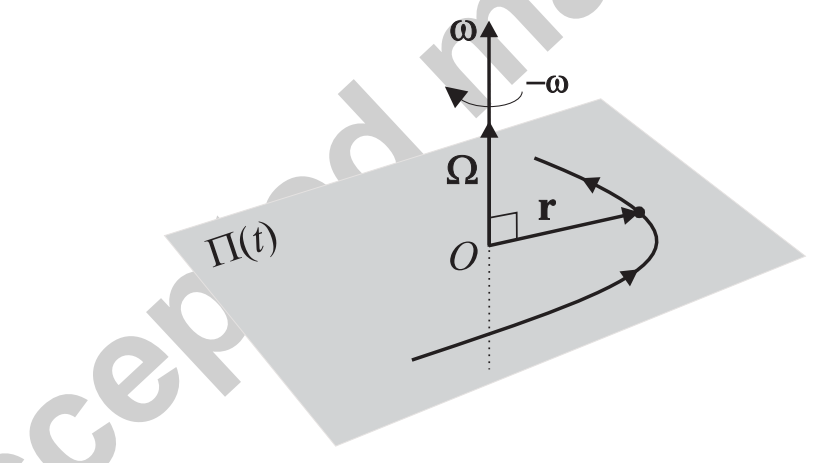

Figure 4: When vector $\boldsymbol{\omega}$ has a fixed direction, that of $\boldsymbol{\Omega}_{0}$, the trajectory is a plane curve.

We conclude that if $\boldsymbol{\Omega}_{0} \neq \mathbf{0}$, the trajectory in the Foucault Pendulum-like problem is a plane curve if the following conditions are satisfied:

1. Vector $\boldsymbol{\omega}$ has a fixed direction;

2. $\boldsymbol{\omega} \times \boldsymbol{\Omega}_{0}=\mathbf{0}$, where $\boldsymbol{\Omega}_{0}=\boldsymbol{\Omega}\left(t_{0}\right)$.

\subsubsection{Zero Generalized Angular Momentum: $\Omega=0$}

The motion may be visualized as described by eq (3.3) on a variable straight line $d(t)$ that rotates with angular velocity $-\boldsymbol{\omega}$ around the fixed point $O$. The vectorial equation of the variable straight line $d(t)$ is given in eq (3.24). The trajectory is a curve situated on a conical surface. This surface is generated by the rotation of $d\left(t_{0}\right)$ with angular velocity $-\boldsymbol{\omega}$ around the fixed point $O$ (see Figure 5).

If vector $\boldsymbol{\omega}$ has a fixed direction, the conical surface becomes a right circular cone. 

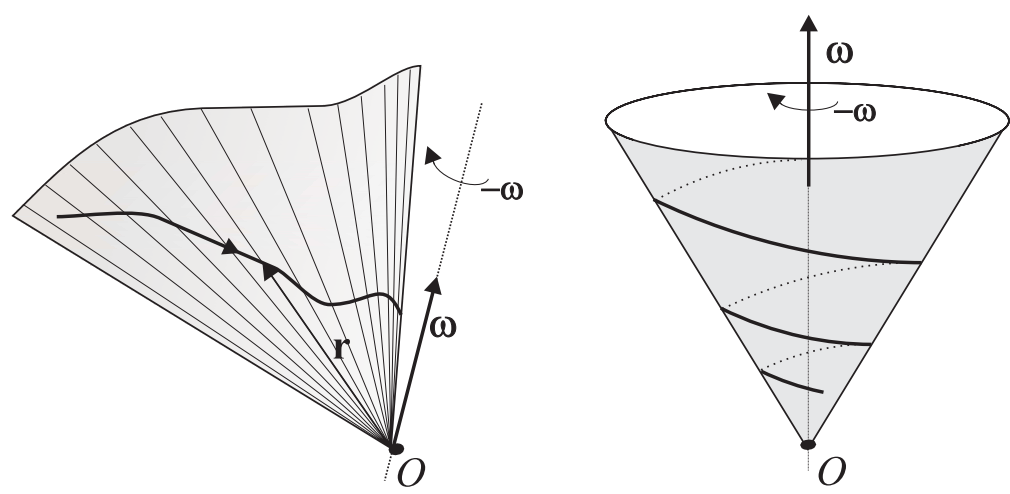

Figure 5: If $\boldsymbol{\Omega}=\mathbf{0}$, the trajectory is situated on a generic conical surface. Left: $\boldsymbol{\omega}$ is arbitrary. Right: $\boldsymbol{\omega}$ has a fixed direction.

\subsection{Law of Motion and Velocity in the Foucault Pendulum-Like Problem}

We have shown that if $\mathbf{r}_{*}$ is the solution to the initial value problem (3.3), the motion described by eq (3.2) is determined by $\mathbf{r}=\mathbf{R}_{-\omega} \mathbf{r}_{*}$. The velocity is computed as: $\mathbf{v}=\dot{\mathbf{r}}=\frac{d}{d t}\left(\mathbf{R}_{-\omega} \mathbf{r}_{*}\right)$. Taking into account eq (2.7), it follows that:

$$
\begin{aligned}
\mathbf{r} & =\mathbf{R}_{-\omega} \mathbf{r}_{*}, \\
\mathbf{v} & =\mathbf{R}_{-\omega} \dot{\mathbf{r}}_{*}-\widetilde{\boldsymbol{\omega}} \mathbf{R}_{-\omega} \mathbf{r}_{*} .
\end{aligned}
$$

If vector $\boldsymbol{\omega}$ has a fixed direction, $\boldsymbol{\omega}=\omega \mathbf{u}$, with $\omega: \mathbb{R} \rightarrow \mathbb{R}$ a differentiable map and $\mathbf{u}$ a constant unit vector, from eqs $(2.8),(3.26),(3.27)$ it results that the law of motion and the velocity may be written as:

$$
\begin{aligned}
\mathbf{r}= & \frac{\boldsymbol{\omega} \cdot \mathbf{r}_{*}}{\omega^{2}} \boldsymbol{\omega}-\frac{\sin \varphi(t)}{\omega} \boldsymbol{\omega} \times \mathbf{r}_{*}-\frac{\cos \varphi(t)}{\omega^{2}} \boldsymbol{\omega} \times\left(\boldsymbol{\omega} \times \mathbf{r}_{*}\right), \\
\mathbf{v}= & \frac{\boldsymbol{\omega} \cdot \dot{\mathbf{r}}_{*}}{\omega^{2}} \boldsymbol{\omega}-\frac{\sin \varphi(t)}{\omega} \boldsymbol{\omega} \times \dot{\mathbf{r}}_{*}-\frac{\cos \varphi(t)}{\omega^{2}} \boldsymbol{\omega} \times\left(\boldsymbol{\omega} \times \dot{\mathbf{r}}_{*}\right) \\
& +\cos \varphi(t) \boldsymbol{\omega} \times \mathbf{r}_{*}-\frac{\sin \varphi(t)}{\omega} \boldsymbol{\omega} \times\left(\boldsymbol{\omega} \times \mathbf{r}_{*}\right),
\end{aligned}
$$

where $\varphi(t)=\int_{t_{0}}^{t} \omega(\xi) d \xi$.

If $\boldsymbol{\omega}$ is constant, then eqs (3.28), (3.29) change by taking $\varphi(t)=\omega\left(t-t_{0}\right)$.

With the help of eqs (3.26) and (3.27), the Foucault Pendulum-like problem is reduced to the study of the initial value problem (3.3).

The study of the motion on the trajectories is splited into two situations, depending on vector $\boldsymbol{\Omega}_{0}$.

\subsubsection{Non-Zero Generalized Angular Momentum: $\Omega \neq 0$}

If $\boldsymbol{\Omega}_{0} \neq \mathbf{0}$, the motion in plane $\boldsymbol{\Pi}(t)$, which is described by the inertial problem (3.3), may be studied using polar coordinates (see Figure 6).

In plane $i$ a rectilinear motion along the (radial) direction of vector $\mathbf{r}: r=r(t), t \geq t_{0}$ and $(i i)$ a rotation around a fixed axis having the direction of $\boldsymbol{\Omega}_{0}$, given by the polar angle $\theta=\theta(t), t \geq t_{0}$.

The polar axis is chosen as follows: it passes through $O$ and it has the direction of vector $\mathbf{r}_{0}$. The real valued maps $r$ and $\theta$ are the solution to the initial value problem (see [29], [32]):

$$
\left\{\begin{array}{l}
\ddot{r}-r \dot{\theta}^{2}+f(r)=0, \\
r^{2} \dot{\theta}=\Omega_{0}, \\
r\left(t_{0}\right)=r_{0} ; \dot{r}(0)=\frac{\mathbf{r}_{0} \cdot \mathbf{v}_{0}}{r_{0}} ; \theta\left(t_{0}\right)=0
\end{array}\right.
$$




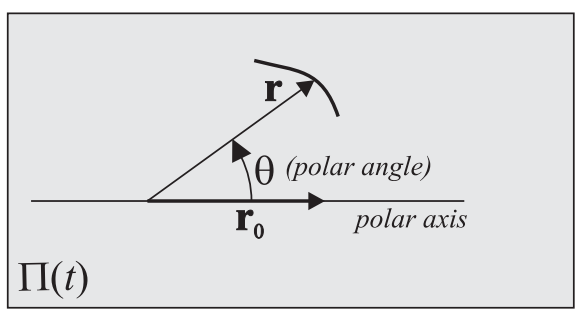

Figure 6: Polar coordinates in plane $\boldsymbol{\Pi}(t)$.

that leads to:

$$
\left\{\begin{array}{l}
\ddot{r}-\frac{\Omega_{0}^{2}}{r^{3}}+f(r)=0 \\
r\left(t_{0}\right)=r_{0} ; \dot{r}(0)=\frac{\mathbf{r}_{0} \cdot \mathbf{v}_{0}}{r_{0}}
\end{array}\right.
$$

Eq (3.31) has the first integral:

$$
\frac{\dot{r}^{2}}{2}+\frac{\Omega_{0}^{2}}{2 r^{2}}+U(r)=\frac{1}{2}\left(\frac{\mathbf{r}_{0} \cdot \mathbf{v}_{0}}{r_{0}}\right)^{2}+\frac{\Omega_{0}^{2}}{2 r_{0}^{2}}+U\left(r_{0}\right) \stackrel{\text { def }}{=} E .
$$

From eq (3.32) it follows that:

$$
t-t_{0}= \pm \int_{r_{0}}^{r} \frac{d \rho}{\sqrt{2\left[E-U_{\text {effective }}(\rho)\right]}}
$$

where:

$$
\left\{\begin{array}{l}
U_{\text {effective }}(\rho) \stackrel{\text { def }}{=} \frac{\Omega_{0}^{2}}{2 \rho^{2}}+U(\rho), \\
U(r)=\int f(r) d r .
\end{array}\right.
$$

The sign "+ " in eq (3.33) is chosen when map $r$ increases and " $-"$ when it decreases (see [29]-[32]). If $r_{*}$ is the solution to eq (3.31) and using the notation:

$$
\underline{\omega} \stackrel{\text { def }}{=} \dot{\theta} \frac{\Omega_{0}}{\Omega_{0}}=\frac{\Omega_{0}}{r_{*}^{2}}
$$

the tensor map $\mathbf{R}_{\underline{\omega}} \in \mathbf{S O}_{3}^{\mathbb{R}}$ may be introduced:

$$
\mathbf{R}_{\underline{\omega}} \stackrel{\text { def }}{=} \mathbf{I}_{3}+\frac{\sin \theta(t)}{\Omega_{0}} \widetilde{\Omega}_{0}+\frac{1-\cos \theta(t)}{\Omega_{0}^{2}} \widetilde{\Omega}_{0}^{2} .
$$

The tensor map $\mathbf{R}_{\underline{\omega}}$ models the rotation with angular velocity $\underline{\omega}=\frac{1}{r_{*}^{2}} \boldsymbol{\Omega}_{0}$. The solution to the initial value problem (3.3) may now be written as:

$$
\mathbf{r}_{*}=r_{*} \mathbf{R}_{\underline{\omega}} \frac{\mathbf{r}_{0}}{r_{0}}
$$

Remark that the map $\theta=\theta(t)$ has the following expressions:

$$
\theta(t)=\Omega_{0} \int_{t_{0}}^{t} \frac{1}{r_{*}^{2}(\tau)} d \tau
$$


By taking into account eq (3.37) and the considerations made in Section $\mathbf{3 . 1}$, we conclude that if $\boldsymbol{\Omega}_{0} \neq \mathbf{0}$, then the law of motion in the Foucault Pendulum-like motion may be written in the following form:

$$
\mathbf{r}=r_{*} \mathbf{R}_{-\boldsymbol{\omega}} \mathbf{R}_{\underline{\omega}} \frac{\mathbf{r}_{0}}{r_{0}}
$$

where: $r_{*}$ is the solution to eq $(3.31) ; \mathbf{R}_{-\boldsymbol{\omega}}$ is the solution to eq $(2.7) ; \mathbf{R}_{\underline{\omega}}$ is given by eq $(3.36)$.

By computing

$$
\dot{\mathbf{R}}_{\underline{\omega}}=\underline{\widetilde{\omega}} \mathbf{R}_{\underline{\omega}}=\mathbf{R}_{\underline{\omega}} \underline{\widetilde{\omega}}
$$

and taking eq (3.36) into account, it follows that the velocity in the Foucault Pendulum-like motion may be written as:

$$
\mathbf{v}=\dot{r}_{*} \mathbf{R}_{-\boldsymbol{\omega}} \mathbf{R}_{\underline{\omega}} \frac{\mathbf{r}_{0}}{r_{0}}+r_{*}\left(\mathbf{R}_{-\omega} \underline{\tilde{\omega}}-\widetilde{\omega} \mathbf{R}_{-\boldsymbol{\omega}}\right) \mathbf{R}_{\underline{\omega}} \frac{\mathbf{r}_{0}}{r_{0}} .
$$

There is a particular remarkable situation if $\boldsymbol{\Omega}_{0} \neq \mathbf{0}$, when the trajectory in plane $\boldsymbol{\Pi}(t)$ is circular. It occurs when:

$$
\left\{\begin{array}{l}
\mathbf{r}_{0} \cdot \mathbf{v}_{0}=0 \\
f\left(r_{0}\right)=\frac{\left(\mathbf{v}_{0}+\omega_{0} \times \mathbf{r}_{0}\right)^{2}}{r_{0}}
\end{array}\right.
$$

as it follows from eq (3.31). The hodograph of the solution to the initial value problem (3.2) is a spherical curve, since $r_{*}$ is constant, $r_{*}=r_{0}$. The radius of the sphere is $r_{0}$ (see Figure 7 ).

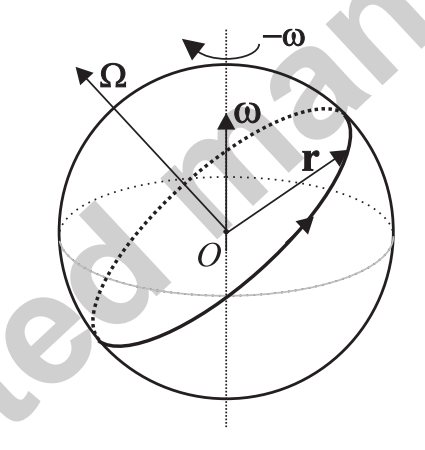

Figure 7: The circular trajectory in plane $\boldsymbol{\Pi}(t)$ determines a spherical trajectory in the Foucault Pendulum-like motion.

We conclude that if $\boldsymbol{\Omega}_{0} \neq \mathbf{0}$, then the law of motion and velocity in the Foucault Pendulum-like problem described by eq (3.2) with the conditions (3.42) are:

$$
\left\{\begin{array}{l}
\mathbf{r}=\mathbf{R}_{-\omega} \mathbf{R}_{\underline{\omega}} \mathbf{r}_{0}, \\
\mathbf{v}=\left(\mathbf{R}_{-\omega} \underline{\tilde{\omega}}-\widetilde{\omega} \mathbf{R}_{-\omega}\right) \mathbf{R}_{\underline{\omega}} \mathbf{r}_{0},
\end{array}\right.
$$

where $\mathbf{R}_{\underline{\omega}}$ models the regular precession with angular velocity $\frac{1}{r_{0}^{2}} \boldsymbol{\Omega}_{0}$ and has the particular expression:

$$
\mathbf{R}_{\underline{\omega}} \stackrel{\text { def }}{=} \mathbf{I}_{3}+\frac{\sin \left[\Omega_{0} r_{0}^{-2}\left(t-t_{0}\right)\right]}{\Omega_{0}} \widetilde{\Omega}_{0}+\frac{1-\cos \left[\Omega_{0} r_{0}^{-2}\left(t-t_{0}\right)\right]}{\Omega_{0}^{2}} \widetilde{\Omega}_{0}^{2} .
$$

\subsubsection{Zero Generalized Angular Momentum: $\Omega=0$}

The solution to the initial value problem (3.2) may be written as in eq (3.39) by taking $\mathbf{R}_{\underline{\omega}}=\mathbf{I}_{3}$ and $r_{*}$ the solution to the initial value problem:

$$
\left\{\begin{array}{l}
\ddot{r}+f(r)=0 \\
r\left(t_{0}\right)=r_{0}, \dot{r}\left(t_{0}\right)=\frac{\mathbf{r}_{0} \cdot \mathbf{v}_{0}}{r_{0}}
\end{array}\right.
$$


From eqs (3.45) it follows that the relation between $r$ and $t$ may be obtained by using eq (3.33) with $\Omega_{0}=0$ (remark that in this case $U_{\text {effective }}(\rho)=U(\rho)$ ), as it follows from eq (3.34).

The law of motion and velocity in the Foucault Pendulum-like problem described by eq (3.2) in the case when $\boldsymbol{\Omega}_{0}=\mathbf{0}$ are expressed below:

$$
\begin{aligned}
\mathbf{r} & =r_{*} \mathbf{R}_{-\boldsymbol{\omega}} \frac{\mathbf{r}_{0}}{r_{0}}, \\
\mathbf{v} & =\left(\dot{r}_{*} \mathbf{R}_{-\boldsymbol{\omega}}-r_{*} \widetilde{\omega} \mathbf{R}_{-\boldsymbol{\omega}}\right) \frac{\mathbf{r}_{0}}{r_{0}},
\end{aligned}
$$

where $r_{*}$ is the solution to eq (3.45).

\subsubsection{Conclusive Remarks}

1. The law of motion and the velocity in the Foucault Pendulum-like problem may be expressed as:

$$
\begin{aligned}
\mathbf{r} & =r_{*} \mathbf{R}_{*} \frac{\mathbf{r}_{0}}{r_{0}}, \\
\mathbf{v} & =\left(\dot{r}_{*} \mathbf{R}_{*}-r_{*} \widetilde{\boldsymbol{\omega}}_{*} \mathbf{R}_{*}\right) \frac{\mathbf{r}_{0}}{r_{0}},
\end{aligned}
$$

where $\mathbf{R}_{*} \in \mathbf{S O}_{3}^{\mathbb{R}}$ is the orthogonal proper tensor that models the rotation with instantaneous angular velocity $\boldsymbol{\omega}_{*}$, where (see [21]):

$$
\boldsymbol{\omega}_{*}=-\boldsymbol{\omega}+\frac{1}{r_{*}^{2}} \mathbf{R}_{-\boldsymbol{\omega}} \boldsymbol{\Omega}_{0},
$$

and $r_{*}$ is the solution to eq (3.31). This method suggests that the study of the solution to the initial value problem (3.2) reduces to the study of the radial motion described by eq (3.31) and the rotation modeled by the tensor map $\mathbf{R}_{*}$ defined above. Eq (3.50) is obtained by using: $\mathbf{R}_{*}=\mathbf{R}_{-\boldsymbol{\omega}} \mathbf{R}_{\underline{\omega}}$.

2. In the particular situation $\mathbf{R}_{-\boldsymbol{\omega}} \boldsymbol{\Omega}_{0}=r_{*}^{2} \boldsymbol{\omega}$, then $\mathbf{R}_{*}=\mathbf{I}_{3}$ and from eq (3.46) it follows that the trajectory of the Foucault Pendulum-like motion is rectilinear.

\subsection{Earth Satellites and the Foucault Pendulum-Like Problem}

Consider a satellite orbiting Earth. Its motion is referred to a rotating reference frame originated in the center of the planet. The motion of the satellite under the influence of the gravitational attraction force is modeled by the initial value problem:

$$
\left\{\begin{array}{l}
\ddot{\mathbf{r}}+2 \boldsymbol{\omega}_{E} \times \dot{\mathbf{r}}+\boldsymbol{\omega}_{E} \times\left(\boldsymbol{\omega}_{E} \times \mathbf{r}\right)+\frac{\mu}{r^{3}} \mathbf{r}=\mathbf{0}, \\
\mathbf{r}\left(t_{0}\right)=\mathbf{r}_{0} ; \dot{\mathbf{r}}\left(t_{0}\right)=\mathbf{v}_{0}
\end{array}\right.
$$

with $\mu>0$ the Earth gravitational parameter. Here $\boldsymbol{\omega}_{E}$ represents the Earth angular velocity. The rotating reference frame has the origin at the center of Earth and rotates with the same angular velocity as the Earth. It is known as the Earth Centered Non-Inertial reference frame (ECNI) (see [54]).

From Section 3.1 it follows that, with respect to the ECNI reference frame, any satellite moves in a variable plane that has a regular precession motion with angular velocity $-\boldsymbol{\omega}_{E}$ (see Figure 8). This precession motion does not depend on the orbital elements of the satellite (see [54] - [56]).

The law of motion may be written as it follows:

$$
\mathbf{r}=\mathbf{R}_{-\omega_{E}} \mathbf{r}_{*},
$$

where $\mathbf{r}_{*}$ is the solution to the initial value problem:

$$
\left\{\begin{array}{l}
\ddot{\mathbf{r}}+\frac{\mu}{r^{3}} \mathbf{r}=\mathbf{0}, \\
\mathbf{r}\left(t_{0}\right)=\mathbf{r}_{0} ; \dot{\mathbf{r}}\left(t_{0}\right)=\mathbf{v}_{0}+\boldsymbol{\omega}_{E} \times \mathbf{r}_{0}
\end{array}\right.
$$




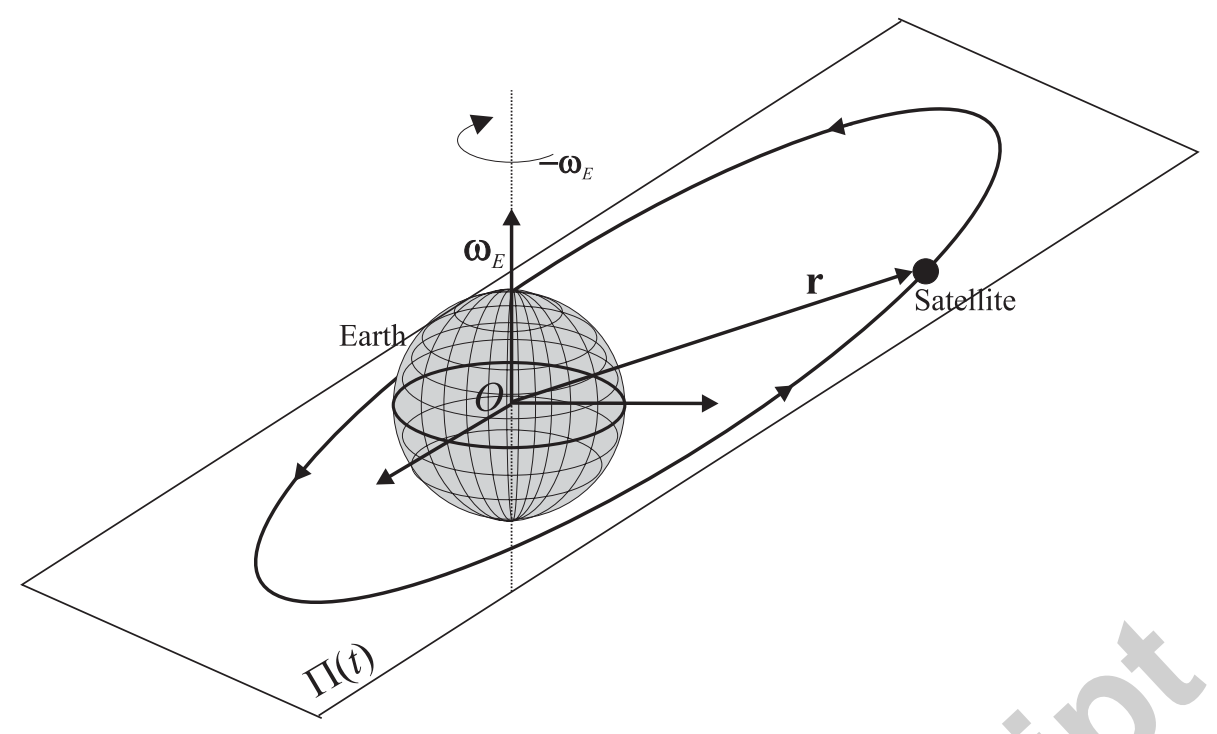

Figure 8: The trajectory of an Earth satellite related to the ECNI reference frame.

Eq (3.53) represents the classic Kepler problem (see [29], [30], [32], [54]).

The closed form exact vectorial expressions for the law of motion and for the velocity may be obtained from here. In the situation when the hodograph of the solution to eq (3.53) is an ellipse (which is the case of an Earth satellite, for example), then (see [21]):

$$
\begin{aligned}
\mathbf{r}(t)= & {[\cos E(t)-e] \mathbf{R}_{-\boldsymbol{\omega}_{E}} \mathbf{a}_{0}+\sin E(t) \mathbf{R}_{-\boldsymbol{\omega}_{E}} \mathbf{b}_{0}, } \\
\mathbf{v}(t)= & \frac{n}{1-e \cos E(t)}\left[-\sin E(t) \mathbf{R}_{-\boldsymbol{\omega}_{E}} \mathbf{a}_{0}+\cos E(t) \mathbf{R}_{-\boldsymbol{\omega}_{E}} \mathbf{b}_{0}\right] \\
& -[\cos E(t)-e]\left[\boldsymbol{\omega}_{E} \times \mathbf{R}_{-\boldsymbol{\omega}_{E}} \mathbf{a}_{0}\right]-\sin E(t)\left[\boldsymbol{\omega}_{E} \times \mathbf{R}_{-\boldsymbol{\omega}_{E}} \mathbf{b}_{0}\right],
\end{aligned}
$$

where

$$
\mathbf{a}_{0}=\left\{\begin{array}{cc}
\frac{\mu}{2 e|h|} \mathbf{e}_{0}, & \mathbf{e}_{0} \neq \mathbf{0} \\
\mathbf{r}_{0}, & \mathbf{e}_{0}=\mathbf{0}
\end{array} ; \quad \mathbf{b}_{0}=\left\{\begin{array}{cc}
\frac{1}{e \sqrt{2|h|} \boldsymbol{\Omega}_{0} \times \mathbf{e}_{0},} & \mathbf{e}_{0} \neq \mathbf{0}, \\
\frac{1}{n}\left(\mathbf{v}_{0}+\boldsymbol{\omega}_{E} \times \mathbf{r}_{0}\right), & \mathbf{e}_{0}=\mathbf{0},
\end{array}\right.\right.
$$

represent the vectorial semimajor and semiminor axes of the elliptic inertial trajectory, $\mathbf{e}_{0}$ represents the vectorial eccentricity of the trajectory and $e$ is its magnitude:

$$
\begin{aligned}
\mathbf{e}_{0} & =\frac{\left(\mathbf{v}_{0}+\boldsymbol{\omega}_{E} \times \mathbf{r}_{0}\right) \times\left[\mathbf{r}_{0} \times\left(\mathbf{v}_{0}+\boldsymbol{\omega}_{E} \times \mathbf{r}_{0}\right)\right]}{\mu}-\frac{\mathbf{r}_{0}}{r_{0}}, \\
e & =\sqrt{1-\frac{2 \Omega_{0}^{2}|h|}{\mu^{2}} ;} \quad n=\frac{(2|h|)^{3 / 2}}{\mu}
\end{aligned}
$$

$t \in\left[t_{0},+\infty\right)$. Here $h$ denotes the specific energy,

$$
h=\frac{1}{2}\left(\mathbf{v}_{0}+\boldsymbol{\omega}_{E} \times \mathbf{r}_{0}\right)^{2}-\frac{\mu}{r_{0}}<0,
$$

and the constant $n$ is named mean motion. Vector $\boldsymbol{\Omega}_{0}$ represents the specific angular momentum of the inertial trajectory:

$$
\boldsymbol{\Omega}_{0}=\mathbf{r}_{0} \times\left(\mathbf{v}_{0}+\boldsymbol{\omega}_{E} \times \mathbf{r}_{0}\right) .
$$

Function $E(t)$ is the eccentric anomaly. It is defined by:

$$
E(t)-e \sin E(t)=n\left(t-t_{0}\right)+E_{0}-e \sin E_{0}, t \in\left[t_{0},+\infty\right),
$$


with $E_{0} \in[0,2 \pi)$ given by:

$$
\cos E_{0}=\frac{1}{e}\left(1-n \frac{r_{0}}{\sqrt{2|h|}}\right), \sin E_{0}=n \frac{\left(\mathbf{v}_{0} \cdot \mathbf{r}_{0}\right)}{2 e|h|}\left[1-\frac{\boldsymbol{\omega}_{E} \cdot \boldsymbol{\Omega}_{0}}{\mu} r_{0}\right] .
$$

By taking into account that tensor $\mathbf{R}_{-\boldsymbol{\omega}_{E}}$ has the explicit expression:

$$
\mathbf{R}_{-\boldsymbol{\omega}_{E}}=\mathbf{I}_{3}-\sin \left[\omega_{E}\left(t-t_{0}\right)\right] \frac{\widetilde{\boldsymbol{\omega}}_{E}}{\omega_{E}}+\left\{1-\cos \left[\omega_{E}\left(t-t_{0}\right)\right]\right\} \frac{\widetilde{\boldsymbol{\omega}}_{E}^{2}}{\omega_{E}^{2}},
$$

it follows that eqs (3.54) may be expressed as:

$$
\begin{aligned}
\mathbf{r}(t)= & {[\cos E(t)-e]\left\{\mathbf{a}_{0}-\sin \left[\omega_{E}\left(t-t_{0}\right)\right] \frac{\boldsymbol{\omega}_{E} \times \mathbf{a}_{0}}{\omega_{E}}+\left\{1-\cos \left[\omega_{E}\left(t-t_{0}\right)\right]\right\} \frac{\boldsymbol{\omega}_{E} \times\left(\boldsymbol{\omega}_{E} \times \mathbf{a}_{0}\right)}{\omega_{E}^{2}}\right\}+} \\
& \sin E(t)\left\{\mathbf{b}_{0}-\sin \left[\omega_{E}\left(t-t_{0}\right)\right] \frac{\boldsymbol{\omega}_{E} \times \mathbf{b}_{0}}{\omega_{E}}+\left\{1-\cos \left[\omega_{E}\left(t-t_{0}\right)\right]\right\} \frac{\boldsymbol{\omega}_{E} \times\left(\boldsymbol{\omega}_{E} \times \mathbf{b}_{0}\right)}{\omega_{E}^{2}}\right\} ; \\
\mathbf{v}(t)= & \frac{-n \sin E(t)}{1-e \cos E(t)}\left\{\mathbf{a}_{0}-\sin \left[\omega_{E}\left(t-t_{0}\right)\right] \frac{\boldsymbol{\omega}_{E} \times \mathbf{a}_{0}}{\omega_{E}}+\left\{1-\cos \left[\omega_{E}\left(t-t_{0}\right)\right]\right\} \frac{\boldsymbol{\omega}_{E} \times\left(\boldsymbol{\omega}_{E} \times \mathbf{a}_{0}\right)}{\omega_{E}^{2}}\right\}+ \\
& \frac{n \cos E(t)}{1-e \cos E(t)}\left\{\mathbf{b}_{0}-\sin \left[\omega_{E}\left(t-t_{0}\right)\right] \frac{\boldsymbol{\omega}_{E} \times \mathbf{b}_{0}}{\omega_{E}}+\left\{1-\cos \left[\omega_{E}\left(t-t_{0}\right)\right]\right\} \frac{\boldsymbol{\omega}_{E} \times\left(\boldsymbol{\omega}_{E} \times \mathbf{b}_{0}\right)}{\omega_{E}^{2}}\right\}- \\
& {[\cos E(t)-e]\left\{\cos \left[\omega_{E}\left(t-t_{0}\right)\right] \boldsymbol{\omega}_{E} \times \mathbf{a}_{0}-\sin \left[\omega_{E}\left(t-t_{0}\right)\right] \frac{\boldsymbol{\omega}_{E} \times\left(\boldsymbol{\omega}_{E} \times \mathbf{a}_{0}\right)}{\omega_{E}}\right\}-} \\
& \sin E(t)\left\{\cos \left[\omega_{E}\left(t-t_{0}\right)\right] \boldsymbol{\omega}_{E} \times \mathbf{b}_{0}-\sin \left[\omega_{E}\left(t-t_{0}\right)\right] \frac{\boldsymbol{\omega}_{E} \times\left(\boldsymbol{\omega}_{E} \times \mathbf{b}_{0}\right)}{\omega_{E}}\right\} .
\end{aligned}
$$

This approach allows to determine the sub-satellite trajectory (the ground-track) (see [54]). Its parametric vectorial equation is:

$$
\mathbf{e}_{\mathbf{R}}=R_{E} \mathbf{R}_{-\omega_{E}} \frac{\mathbf{r}_{*}}{r_{*}},
$$

where $R_{E}$ is the Earth radius and $\mathbf{r}_{*}$ is the solution to the initial value problem (3.53). The exact vectorial expression for $\mathbf{e}_{\mathbf{R}}$ is (see also [22]):

$$
\begin{aligned}
\mathbf{e}_{\mathbf{R}}= & \frac{R_{E}[\cos E(t)-e]}{a_{0}[1-e \cos E(t)]}\left\{\mathbf{a}_{0}-\sin \left[\omega_{E}\left(t-t_{0}\right)\right] \frac{\boldsymbol{\omega}_{E} \times \mathbf{a}_{0}}{\omega_{E}}+\left\{1-\cos \left[\omega_{E}\left(t-t_{0}\right)\right]\right\} \frac{\boldsymbol{\omega}_{E} \times\left(\boldsymbol{\omega}_{E} \times \mathbf{a}_{0}\right)}{\omega_{E}^{2}}\right\}+ \\
& \frac{R_{E} \sin E(t)}{a_{0}[1-e \cos E(t)]}\left\{\mathbf{b}_{0}-\sin \left[\omega_{E}\left(t-t_{0}\right)\right] \frac{\boldsymbol{\omega}_{E} \times \mathbf{b}_{0}}{\omega_{E}}+\left\{1-\cos \left[\omega_{E}\left(t-t_{0}\right)\right]\right\} \frac{\boldsymbol{\omega}_{E} \times\left(\boldsymbol{\omega}_{E} \times \mathbf{b}_{0}\right)}{\omega_{E}^{2}}\right\} .
\end{aligned}
$$

To the knowledge of the authors, this Foucault Pendulum-like motion of an Earth satellite was not pointed out until now.

\subsection{The Keplerian Relative Orbital Motion is a Generalized Foucault Pendulum-Like Motion}

The relative orbital motion study became of great interest in the recent years, because to its applications in present and future space missions that involve satellite flying formations, satellite constellations and orbital rendezvous. Numerous papers were written especially in the past few years (see [10], [12] - [26], [37] - [41]), but the starting point in this field consists of the results obtained by Clohessy and Wiltshire [6], Lawden [7], Tschauner and Hempel [8, 9].

Consider two satellites orbiting under the influence of the same attraction center (see Figure 9). One of them is considered to be the main satellite, it will be named Chief and its trajectory will be named reference trajectory. The other satellite is usually named Deputy and its trajectory is known as the targeted trajectory. The main problem is to study the motion of the Deputy with respect to a non-inertial reference frame originated in the center of mass $C$ of the Chief. This reference frame is traditionally chosen so that the $C x$ axis have the same orientation as the position vector of the Chief with respect to an inertial reference frame originated in the attraction center, the $C z$ axis have the same orientation as the angular momentum of the Chief and the $C y$ axis complete a right-handed 


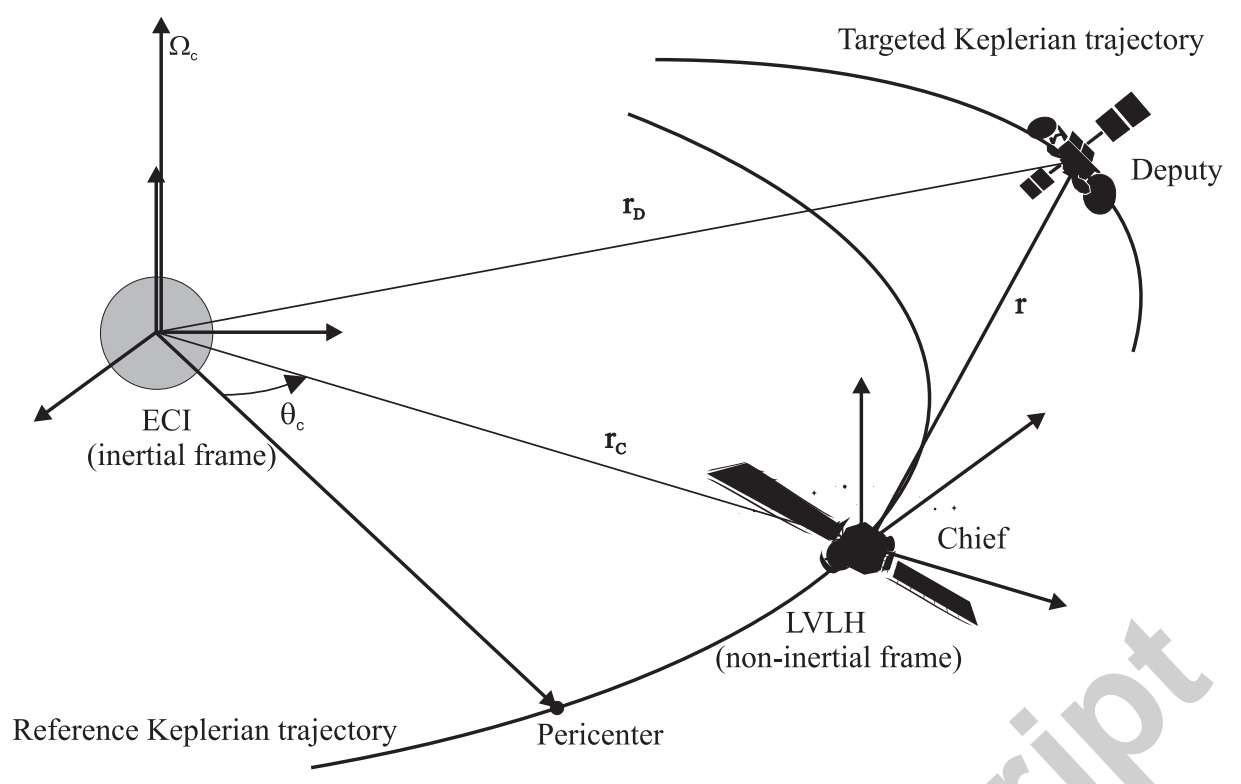

Figure 9: Relative orbital motion: physical model.

frame. Its name is LVLH (Local-Vertical-Local-Horizontal) (see [54]). An inertial reference frame is also considered in this model. It has the origin in the Earth center and it will be named ECI (Earth Centered Inertial) (see [54]).

Consider $\boldsymbol{\omega}=\boldsymbol{\omega}(t)$ the angular velocity of the LVLH reference frame with respect to an inertial frame originated in the attraction center. By denoting $\mathbf{r}_{c}$ the Chief position vector with respect to an inertial frame originated in $O$ (the attraction center), $\theta_{c}=\theta_{c}(t)$ the true anomaly, $e_{c}$ the eccentricity, $\boldsymbol{\Omega}_{c}$ the Chief orbit specific angular momentum and $p_{c}$ the semilatus rectum of the Chief orbit, it follows that vector $\boldsymbol{\omega}$ has the expression:

$$
\boldsymbol{\omega}=\dot{\theta}_{c} \frac{\boldsymbol{\Omega}_{c}}{\Omega_{c}}==\frac{1}{r_{c}^{2}} \boldsymbol{\Omega}_{c}=\left[\frac{1+e_{c} \cos \theta_{c}(t)}{p_{c}}\right]^{2} \boldsymbol{\Omega}_{c},
$$

where vector $\mathbf{r}_{c}$ is expressed with respect to the LVLH frame and has the form:

$$
\mathbf{r}_{c}=\frac{p_{c}}{1+e_{c} \cos \theta_{c}(t)} \frac{\mathbf{r}_{0}}{r_{0}} .
$$

Vector $\mathbf{r}_{0}$ points to the initial position of the Chief satellite with respect to the inertial reference frame originated in the attraction center $O$. The initial value problem that models the motion of the Deputy satellite with respect to the LVLH reference frame is (see [24], [26], [54]):

$$
\left\{\begin{array}{l}
\ddot{\mathbf{r}}+\mathbf{2} \boldsymbol{\omega} \times \dot{\mathbf{r}}+\boldsymbol{\omega} \times(\boldsymbol{\omega} \times \mathbf{r})+\dot{\boldsymbol{\omega}} \times \mathbf{r}+\frac{\mu}{\left|\mathbf{r}_{c}+\mathbf{r}\right|^{3}}\left(\mathbf{r}_{c}+\mathbf{r}\right)-\frac{\mu}{r_{c}^{3}} \mathbf{r}_{c}=\mathbf{0}, \\
\mathbf{r}\left(t_{0}\right)=\Delta \mathbf{r}, \dot{\mathbf{r}}\left(t_{0}\right)=\Delta \mathbf{v}
\end{array}\right.
$$

where $\mu>0$ is the gravitational parameter of the attraction center and $\Delta \mathbf{r}, \Delta \mathbf{v}$ represent the relative position and relative velocity vectors of the Deputy satellite with respect to LVLH at the initial moment of time $t_{0} \geq 0$.

Most studies start from the linearized form of eq (3.68), which is obtained from eq (3.68) by making the assumtion that the distance netween the two satellites remains small relative to the distance to the attraction centre, more exactly that: $\left(r / r_{c}\right)^{2} \simeq 0$. Then eq (3.68) may be written in the linearized form:

$$
\left\{\begin{array}{l}
\ddot{\mathbf{r}}+\mathbf{2} \boldsymbol{\omega} \times \dot{\mathbf{r}}+\boldsymbol{\omega} \times(\boldsymbol{\omega} \times \mathbf{r})+\dot{\boldsymbol{\omega}} \times \mathbf{r}+\omega^{2} \mathbf{r}=3 \omega^{2} \frac{\mathbf{r} \cdot \mathbf{r}_{c}}{r_{c}^{2}} \mathbf{r}_{c} \\
\mathbf{r}\left(t_{0}\right)=\Delta \mathbf{r}, \dot{\mathbf{r}}\left(t_{0}\right)=\Delta \mathbf{v}
\end{array}\right.
$$

Eq (3.68) represents the vectorial form of the Hill equation (see $[10,57]$ ). Since the celebrated results of Clohessy and Wiltshire (see [6]) in the early 1960's, many attempts to solve this equation were made. In [6], the authors offer 
a solution to the linearized equation (3.69) in the particular case when the reference trajectory is circular. The main assumption made in their approach is that the relative distance remains small with respect to the distance to the attraction center of the Chief satellite during the entire motion. The same assumption was made by Lawden (see [7]) and Tschauner and Hempel (see $[8,9]$ ) when they offered a solution to the linearized equation (3.69) in the particular case when the reference trajectory is elliptic. Both solutions (in the case of circular and elliptic reference trajectories) contain secular terms or singularities. These singularities were eliminated in several attempts made by Carter (see [11]) and Sengupta (see [12]), and closed form expressions for the solution to eq (3.69) were obtained. Other approaches to the linearized equations of motion use a classic concept in the theory of linear systems: the state transition matrix (see $[13,14])$. The nonlinear model (3.68) is studied in more recent papers. Balaji and Tatnall (see [15]) Gurfil and Kasdin (see [16]) and other authors (see [17, 18, 19]) offer the solution to the nonlinear model by geometric procedures, in the particular case when both trajectories are elliptic and by using Carthesian coordinates. Gurfil and Kholshevnikov (see [20]) also introduce a metric for the relative motion manifold.

In fact, the solution to eq (3.68) may be seen as a difference between two solutions to two separate initial value problems, both being Foucault Pendulum-like problems. One problem is:

$$
\left\{\begin{array}{l}
\ddot{\mathbf{r}}+\boldsymbol{2} \boldsymbol{\omega} \times \dot{\mathbf{r}}+\boldsymbol{\omega} \times(\boldsymbol{\omega} \times \mathbf{r})+\dot{\boldsymbol{\omega}} \times \mathbf{r}+\frac{\mu}{r^{3}} \mathbf{r}=\mathbf{0}, \\
\mathbf{r}\left(t_{0}\right)=\mathbf{r}_{0}+\Delta \mathbf{r}, \dot{\mathbf{r}}\left(t_{0}\right)=\frac{\mathbf{v}_{0} \cdot \mathbf{r}_{0}}{r_{0}^{2}} \mathbf{r}_{0}+\Delta \mathbf{v},
\end{array}\right.
$$

and the other one is:

$$
\left\{\begin{array}{l}
\ddot{\mathbf{r}}+\mathbf{2} \boldsymbol{\omega} \times \dot{\mathbf{r}}+\boldsymbol{\omega} \times(\boldsymbol{\omega} \times \mathbf{r})+\dot{\boldsymbol{\omega}} \times \mathbf{r}+\frac{\mu}{r^{3}} \mathbf{r}=\mathbf{0}, \\
\mathbf{r}\left(t_{0}\right)=\mathbf{r}_{0}, \dot{\mathbf{r}}\left(t_{0}\right)=\frac{\mathbf{v}_{0} \cdot \mathbf{r}_{0}}{r_{0}^{2}} \mathbf{r}_{0} .
\end{array}\right.
$$

By using the previous remark, the authors of the present paper proved that the solution to the initial value problem (3.68) may be expressed in a vectorial closed form (see $[21,22,24,26])$ :

$$
\left\{\begin{array}{l}
\mathbf{r}=\mathbf{R}_{-\omega} \mathbf{r}_{*}-\mathbf{r}_{c} \\
\mathbf{v}=\mathbf{R}_{-\omega} \dot{\mathbf{r}}_{*}-\widetilde{\omega} \mathbf{R}_{-\omega} \mathbf{r}_{*}-\dot{\mathbf{r}}_{c},
\end{array}\right.
$$

where $\mathbf{r}_{*}$ is the solution to the initial value problem:

$$
\left\{\begin{array}{l}
\ddot{\mathbf{r}}+\frac{\mu}{r^{3}} \mathbf{r}=\mathbf{0}, \\
\mathbf{r}\left(t_{0}\right)=\mathbf{r}_{0}+\Delta \mathbf{r}, \dot{\mathbf{r}}\left(t_{0}\right)=\mathbf{v}_{0}+\Delta \mathbf{v}+\boldsymbol{\omega}_{0} \times \Delta \mathbf{r}
\end{array}\right.
$$

$\left(\mathbf{r}_{c}=\frac{p_{c}}{1+e_{c} \cos \theta_{c}(t)} \frac{\mathbf{r}_{0}}{r_{0}}, \dot{\mathbf{r}}_{c}=\frac{\Omega_{c} e_{c} \sin \theta_{c}(t)}{p_{c}} \frac{\mathbf{r}_{0}}{r_{0}}, \mathbf{r}_{0}, \mathbf{v}_{0}\right.$ represent the initial position and velocity vectors of the Chief with respect to an inertial reference frame originated in the attraction center, and $\left.\boldsymbol{\omega}_{0}=\boldsymbol{\omega}\left(t_{0}\right)=\frac{1}{r_{0}^{2}} \boldsymbol{\Omega}_{c}\right)$. Since vector $\boldsymbol{\omega}$ has constant direction, tensor $\mathbf{R}_{-\boldsymbol{\omega}}$ may be expressed as:

$$
\mathbf{R}_{-\boldsymbol{\omega}}=\mathbf{I}_{3}-\left[\sin \left(\theta_{c}(t)-\theta_{c}\left(t_{0}\right)\right)\right] \frac{\widetilde{\boldsymbol{\Omega}}_{c}}{\Omega_{c}}+\left[1-\cos \left(\theta_{c}(t)-\theta_{c}\left(t_{0}\right)\right)\right] \frac{\widetilde{\boldsymbol{\Omega}}_{c}^{2}}{\Omega_{c}^{2}} .
$$

Exact closed form vectorial expressions for the laws of the relative motion and the relative velocity may be deduced (see $[23,27])$ from eqs $(3.72),(3.74)$ in any situation that may occur (eqs (3.72) are true for any reference or targeted trajectories):

$$
\begin{aligned}
\mathbf{r}= & \frac{\boldsymbol{\Omega}_{c} \cdot \mathbf{r}_{*}}{\boldsymbol{\Omega}_{c}^{2}} \mathbf{h}_{c}-\sin \theta_{c}^{0}(t) \frac{\widetilde{\boldsymbol{\Omega}}_{c} \mathbf{r}_{*}}{\boldsymbol{\Omega}_{c}}-\cos \theta_{c}^{0}(t) \frac{\widetilde{\boldsymbol{\Omega}}_{c}^{2} \mathbf{r}_{*}}{\boldsymbol{\Omega}_{c}^{2}}-\frac{p_{c}}{1+e_{c} \cos \theta_{c}(t)} \frac{\mathbf{r}_{0}}{r_{0}} ; \\
\mathbf{v}= & \frac{\boldsymbol{\Omega}_{c} \cdot \dot{\mathbf{r}}_{*}}{\boldsymbol{\Omega}_{c}^{2}} \mathbf{h}_{c}-\sin \theta_{c}^{0}(t) \frac{\widetilde{\boldsymbol{\Omega}}_{c} \dot{\mathbf{r}}_{*}}{\boldsymbol{\Omega}_{c}}-\cos \theta_{c}^{0}(t) \frac{\widetilde{\boldsymbol{\Omega}}_{c}^{2} \dot{\mathbf{r}}_{*}}{\boldsymbol{\Omega}_{c}^{2}}-\frac{h_{c}\left(1+e_{c} \cos \theta_{c}(t)\right)^{2}}{p_{c}^{2}}\left\{\cos \theta_{c}^{0}(t) \frac{\widetilde{\boldsymbol{\Omega}}_{c} \mathbf{r}_{*}}{\boldsymbol{\Omega}_{c}}\right. \\
& \left.-\sin \theta_{c}^{0}(t) \frac{\widetilde{\boldsymbol{\Omega}}_{c}^{2} \mathbf{r}_{*}}{\boldsymbol{\Omega}_{c}^{2}}\right\}-\frac{\boldsymbol{\Omega}_{c} e_{c} \sin \theta_{c}(t)}{p_{c}} \frac{\mathbf{r}_{0}}{r_{0}}
\end{aligned}
$$


where $\theta_{c}^{0}(t) \stackrel{\text { def }}{=} \theta_{c}(t)-\theta_{c}\left(t_{0}\right)$.

A comprehensive approach to the relative orbital motion may be found in [26]. For a circular reference trajectory, a closed form exact solution to eq (3.68) may be found in [22]. For an arbitrary Keplerian trajectory, an exact closed form solution to eq (3.68) may be found in $[24,26]$.

The first equation in eqs (3.72) leads to a very suggestive geometrical visualization of the Keplerian relative orbital motion. It shows that it is a composition among $(i)$ a classic Keplerian motion in a variable plane $\Pi(t), t \geq t_{0}$; plane $\Pi(t)$ is formed at moment $t=t_{0}$ if the inertial motion of the Deputy satellite is not rectilinear; (ii) a precession of plane $\Pi(t)$ with angular velocity $-\boldsymbol{\omega}$ around the attraction center; (iii) a rectilinear translation of plane $\Pi(t)$ described by vector $-\mathbf{r}_{c}$ (see Figure 10)

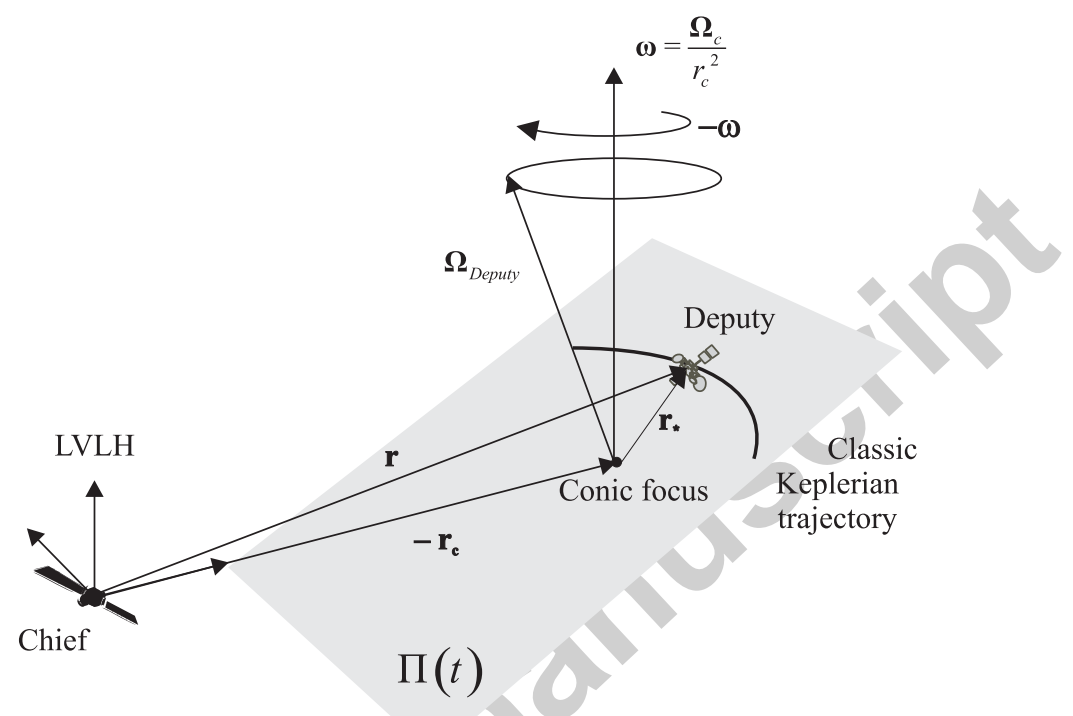

Figure 10: Geometrical vizualisation of the relative Keplerian orbital motion.

The composition of first two motions leads to a Foucault Pendulum-like motion; so we have proven that the relative motion of a satellite with respect to a reference frame originated on another satellite orbiting around the same attraction center is a composition among a Foucault Pendulum-like motion and a rectilinear translation, which we named Generalized Foucault Pendulum-like motion.

\section{A Closed Form Solution to the Foucault Pendulum Problem}

The results from Section 3 will be particularized in order to give an exact vectorial solution to the classic Foucault Pendulum problem described by eq (1.2). As it follows from Section 3.1, the solution to the initial value problem (1.2) is obtained by applying the tensor map $\mathbf{R}_{-\boldsymbol{\omega}}$ to the solution to an inertial initial value problem. The motion in the classic Foucault Pendulum problem is a particular situation of the motion in a central force field with respect to a rotating reference frame originated in the attraction/repulsion center.

In the case of the Foucault Pendulum motion, we state that the solution to the initial value problem:

$$
\left\{\begin{array}{l}
\ddot{\mathbf{r}}+2 \boldsymbol{\omega} \times \dot{\mathbf{r}}+\boldsymbol{\omega} \times(\boldsymbol{\omega} \times \mathbf{r})+\dot{\boldsymbol{\omega}} \times \mathbf{r}+\omega_{*}^{2} \mathbf{r}=\mathbf{0} \\
\mathbf{r}\left(t_{0}\right)=\mathbf{r}_{0} ; \dot{\mathbf{r}}\left(t_{0}\right)=\mathbf{v}_{0}
\end{array}\right.
$$

is obtained by applying the tensor map $\mathbf{R}_{-\boldsymbol{\omega}}$ to the solution to the initial value problem:

$$
\left\{\begin{array}{l}
\ddot{\mathbf{r}}+\omega_{*}^{2} \mathbf{r}=\mathbf{0} \\
\mathbf{r}\left(t_{0}\right)=\mathbf{r}_{0} ; \dot{\mathbf{r}}\left(t_{0}\right)=\mathbf{v}_{0}+\boldsymbol{\omega}_{0} \times \mathbf{r}_{0}
\end{array}\right.
$$

It is a consequence of the considerations made in Section 3.1. The solution to the initial value problem (4.2) is:

$$
\mathbf{r}(t)=\mathbf{r}_{0} \cos \omega_{*}\left(t-t_{0}\right)+\frac{\mathbf{v}_{0}+\boldsymbol{\omega}_{0} \times \mathbf{r}_{0}}{\omega_{*}} \sin \omega_{*}\left(t-t_{0}\right)
$$


Eq (4.2) models a spatial harmonic oscillator. From here we arrive very quickly to the solution to the classic Foucault Pendulum problem (as it is only mentioned in Theoretical Mechanics textbooks). The solution to the initial value problem:

$$
\left\{\begin{array}{l}
\ddot{\mathbf{r}}+2 \boldsymbol{\omega} \times \dot{\mathbf{r}}+\boldsymbol{\omega} \times(\boldsymbol{\omega} \times \mathbf{r})+\dot{\boldsymbol{\omega}} \times \mathbf{r}+\omega_{*}^{2} \mathbf{r}=\mathbf{0} \\
\mathbf{r}\left(t_{0}\right)=\mathbf{r}_{0} ; \dot{\mathbf{r}}\left(t_{0}\right)=\mathbf{v}_{0}
\end{array}\right.
$$

$\left(\boldsymbol{\omega}=\boldsymbol{\omega}(t)\right.$, arbitrary, and $\left.\boldsymbol{\omega}_{0}=\boldsymbol{\omega}\left(t_{0}\right)\right)$, is:

$$
\mathbf{r}(t)=\left(\mathbf{R}_{-\boldsymbol{\omega}} \mathbf{r}_{0}\right) \cos \omega_{*}\left(t-t_{0}\right)+\frac{1}{\omega_{*}} \mathbf{R}_{-\boldsymbol{\omega}}\left(\mathbf{v}_{0}+\boldsymbol{\omega}_{0} \times \mathbf{r}_{0}\right) \sin \omega_{*}\left(t-t_{0}\right) .
$$

If $\mathbf{r}_{0} \times\left(\mathbf{v}_{0}+\boldsymbol{\omega}_{0} \times \mathbf{r}_{0}\right) \neq \mathbf{0}$, the hodograph of the solution to the initial value problem (4.2) is an ellipse. Vectors $\mathbf{r}_{0}$ and $\frac{\mathbf{v}_{0}+\boldsymbol{\omega}_{0} \times \mathbf{r}_{0}}{\omega_{*}}$ are the ellipse conjugate semi-diameters (see [49]). If they are collinear, the ellipse degenerates into a line segment.

Despite the statements in most Theoretical Mechanics textbooks, the closed form solution to the Foucault Pendulum exists and it is given in eq (4.5). It is in fact a harmonic planar oscillation composed with a precession with $-\boldsymbol{\omega}$ angular velocity. Explicit solutions to the Foucault Pendulum may be now derived for two particular cases: vector $\boldsymbol{\omega}$ has constant direction, respectively vector $\boldsymbol{\omega}$ is constant. They are deduced from the explicit expressions of $\mathbf{R}_{-\boldsymbol{\omega}}$ in eqs (2.8) and (2.9), as well as from eq (4.5).

1. If vector $\boldsymbol{\omega}$ has constant direction, $\boldsymbol{\omega}(t)=\omega(t) \mathbf{u}$, with $\mathbf{u}$ its constant unit vector and $\omega:\left[t_{0},+\infty\right) \rightarrow \mathbb{R}$, the solution to the initial value problem (4.1) is:

$$
\begin{aligned}
\mathbf{r}(t)= & \cos \omega_{*}\left(t-t_{0}\right)\left\{\frac{\mathbf{r}_{0} \cdot \boldsymbol{\omega}}{\omega^{2}} \boldsymbol{\omega}-\frac{\sin \varphi(t)}{\omega} \widetilde{\boldsymbol{\omega}} \mathbf{r}_{0}-\frac{\cos \varphi(t)}{\omega^{2}} \widetilde{\boldsymbol{\omega}}^{2} \mathbf{r}_{0}\right\}+ \\
& +\frac{\sin \omega_{*}\left(t-t_{0}\right)}{\omega_{*}}\left\{\frac{\mathbf{v}_{0} \cdot \boldsymbol{\omega}}{\omega^{2}} \boldsymbol{\omega}-\frac{\sin \varphi(t)}{\omega} \widetilde{\boldsymbol{\omega}}\left(\mathbf{v}_{0}+\boldsymbol{\omega}_{0} \times \mathbf{r}_{0}\right)\right\}- \\
& -\frac{\sin \omega_{*}\left(t-t_{0}\right)}{\omega_{*}} \frac{\cos \varphi(t)}{\omega^{2}} \widetilde{\boldsymbol{\omega}}^{2}\left(\mathbf{v}_{0}+\boldsymbol{\omega}_{0} \times \mathbf{r}_{0}\right),
\end{aligned}
$$

where $\varphi(t)=\int_{t_{0}}^{t} \omega(\xi) d \xi$.

2. If vector $\boldsymbol{\omega}$ is constant, the solution to the initial value problem (4.1) is:

$$
\begin{aligned}
\mathbf{r}(t)= & \cos \omega_{*}\left(t-t_{0}\right)\left\{\frac{\mathbf{r}_{0} \cdot \boldsymbol{\omega}}{\omega^{2}} \boldsymbol{\omega}-\frac{\sin \left[\omega\left(t-t_{0}\right)\right]}{\omega} \widetilde{\boldsymbol{\omega}} \mathbf{r}_{0}-\frac{\cos \left[\omega\left(t-t_{0}\right)\right]}{\omega^{2}} \widetilde{\boldsymbol{\omega}}^{2} \mathbf{r}_{0}\right\}+ \\
& +\frac{\sin \omega_{*}\left(t-t_{0}\right)}{\omega_{*}}\left\{\frac{\mathbf{v}_{0} \cdot \boldsymbol{\omega}}{\omega^{2}} \boldsymbol{\omega}-\frac{\sin \left[\omega\left(t-t_{0}\right)\right]}{\omega} \widetilde{\boldsymbol{\omega}}\left(\mathbf{v}_{0}+\boldsymbol{\omega} \times \mathbf{r}_{0}\right)\right\}- \\
& -\frac{\sin \omega_{*}\left(t-t_{0}\right)}{\omega_{*}} \frac{\cos \left[\omega\left(t-t_{0}\right)\right]}{\omega^{2}} \widetilde{\boldsymbol{\omega}}^{2}\left(\mathbf{v}_{0}+\boldsymbol{\omega} \times \mathbf{r}_{0}\right) .
\end{aligned}
$$

Some interesting remarks are to be made here.

1. Eq (4.6) may be rewritten as:

$$
\mathbf{r}(t)=\frac{\boldsymbol{\omega} \cdot \boldsymbol{\Phi}_{*}}{\omega^{2}} \boldsymbol{\omega}-\frac{\sin \varphi(t)}{\omega} \boldsymbol{\omega} \times \boldsymbol{\Phi}_{*}-\frac{\cos \varphi(t)}{\omega^{2}} \boldsymbol{\omega} \times\left(\boldsymbol{\omega} \times \boldsymbol{\Phi}_{*}\right),
$$

where:

$$
\boldsymbol{\Phi}_{*}=\mathbf{r}_{0} \cos \omega_{*}\left(t-t_{0}\right)+\frac{\mathbf{v}_{0}+\omega_{0} \times \mathbf{r}_{0}}{\omega_{*}} \sin \omega_{*}\left(t-t_{0}\right)
$$

2. When vector $\boldsymbol{\omega}$ has constant direction, the motion may be visualized as follows: 
(a) a harmonic oscillation described by eq (4.3) in a variable plane (or along a straight line) that has a fixed point.

(b) a precession of the variable plane (or of the straight line) with angular velocity $-\boldsymbol{\omega}$.

3. Eq (4.7) may be rewritten as:

$$
\mathbf{r}(t)=\frac{\boldsymbol{\omega} \cdot \mathbf{\Phi}_{*}}{\omega^{2}} \boldsymbol{\omega}-\frac{\sin \left[\omega\left(t-t_{0}\right)\right]}{\omega} \boldsymbol{\omega} \times \boldsymbol{\Phi}_{*}-\frac{\cos \left[\omega\left(t-t_{0}\right)\right]}{\omega^{2}} \boldsymbol{\omega} \times\left(\boldsymbol{\omega} \times \mathbf{\Phi}_{*}\right),
$$

where $\boldsymbol{\Phi}_{*}$ is given in eq (4.9).

4. When vector $\boldsymbol{\omega}$ is constant, the motion may be visualized as follows:

(a) an elliptic oscillation described by eq (4.3) in a variable plane (or on a line segment) which has a fixed point;

(b) a regular precession with angular velocity $-\boldsymbol{\omega}$ of the variable plane (or of the line segment).

\subsection{The Trajectories of the Foucault Pendulum Motion}

The study from Section 3.3 is particularized for the Foucault Pendulum trajectories. The motion may be visualized as an elliptic oscillation in a variable plane that rotates with angular velocity $-\boldsymbol{\omega}$ around a fixed point (see Figure 11).

\subsubsection{Non-Zero Generalized Angular Momentum: $\Omega \neq 0$}

The oscillation takes place in a variable plane $\Pi(t), t \geq t_{0}$, that has the normal vector $\boldsymbol{\Omega}$ defined in eq (3.7). This plane rotates with angular velocity $\boldsymbol{-} \boldsymbol{\omega}$ and has a fixed point. If vector $\boldsymbol{\omega}$ has constant direction, the motion of this plane is a precession with angular velocity $-\boldsymbol{\omega}$.

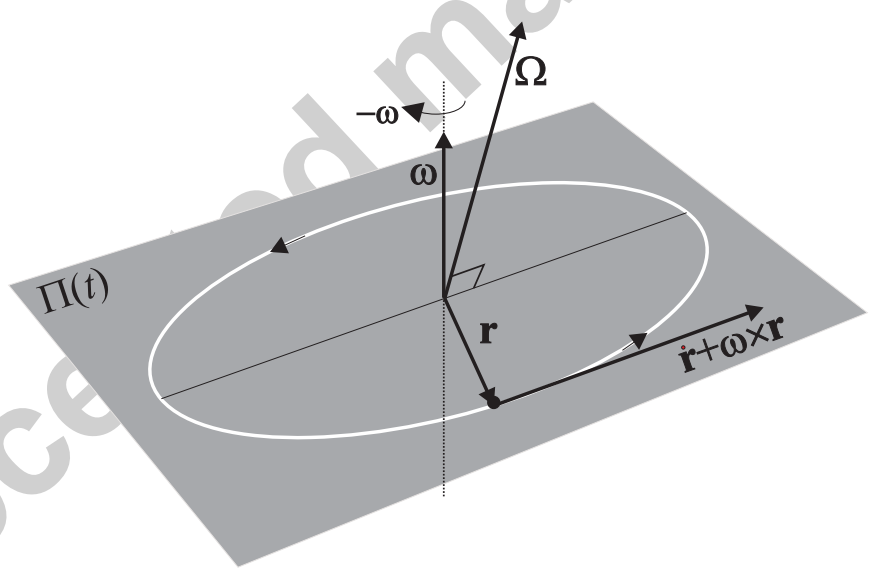

Figure 11: The motion of the Foucault Pendulum.

The vectorial equation of the variable plane $\Pi(t)$ is $\mathbf{r} \cdot \boldsymbol{\Omega}=0, O \in \Pi(t)$, where $O$ is the origin of the rotating reference frame.

With respect to an observatory for whom plane $\boldsymbol{\Pi}(t)$ is fixed, the trajectory is an ellipse. This trajectory has the parametric vectorial equation given by eq (4.3). By denoting:

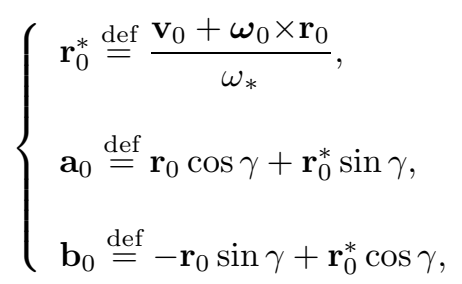


the law of motion related to plane $\boldsymbol{\Pi}(t)$ may be rewritten as (see Appendix):

$$
\mathbf{r}(t)=\mathbf{a}_{0} \cos \left[\omega_{*}\left(t-t_{0}\right)-\gamma\right]+\mathbf{b}_{0} \sin \left[\omega_{*}\left(t-t_{0}\right)-\gamma\right],
$$

where $\mathbf{a}_{0}$ and $\mathbf{b}_{0}$ are the vectorial semiaxes of the ellipse defined in eqs (4.11) and $\gamma \in[0, \pi)$ is uniquely defined by:

$$
\left\{\begin{array}{l}
\cos 2 \gamma=\frac{\mathbf{r}_{0}^{2}-\mathbf{r}_{0}^{* 2}}{\sqrt{\left(\mathbf{r}_{0}^{2}-\mathbf{r}_{0}^{* 2}\right)^{2}+4\left(\mathbf{r}_{0} \cdot \mathbf{r}_{0}^{*}\right)^{2}}} \\
\sin 2 \gamma=\frac{2\left(\mathbf{r}_{0} \cdot \mathbf{r}_{0}^{*}\right)}{\sqrt{\left(\mathbf{r}_{0}^{2}-\mathbf{r}_{0}^{* 2}\right)^{2}+4\left(\mathbf{r}_{0} \cdot \mathbf{r}_{0}^{*}\right)^{2}}}
\end{array}\right.
$$

when $\left(\mathbf{r}_{0}^{2}-\mathbf{r}_{0}^{* 2}\right)^{2}+4\left(\mathbf{r}_{0} \cdot \mathbf{r}_{0}^{*}\right)^{2} \neq 0$. If $\left(\mathbf{r}_{0}^{2}-\mathbf{r}_{0}^{* 2}\right)^{2}+4\left(\mathbf{r}_{0} \cdot \mathbf{r}_{0}^{*}\right)^{2}=0$, the motion in plane $\boldsymbol{\Pi}(t)$ is a uniform circular one, with the vectorial parametric law of motion:

$$
\mathbf{r}(t)=\mathbf{r}_{0} \cos \left[\omega_{*}\left(t-t_{0}\right)\right]+\mathbf{r}_{0}^{*} \sin \left[\omega_{*}\left(t-t_{0}\right)\right] .
$$

The Foucault Pendulum trajectory is generally a space curve, bounded between two concentric spheres, one with radius $a$ and the other with radius $b$, where $a$ and $b$ represent the ellipse semiaxes (see Figure 12).

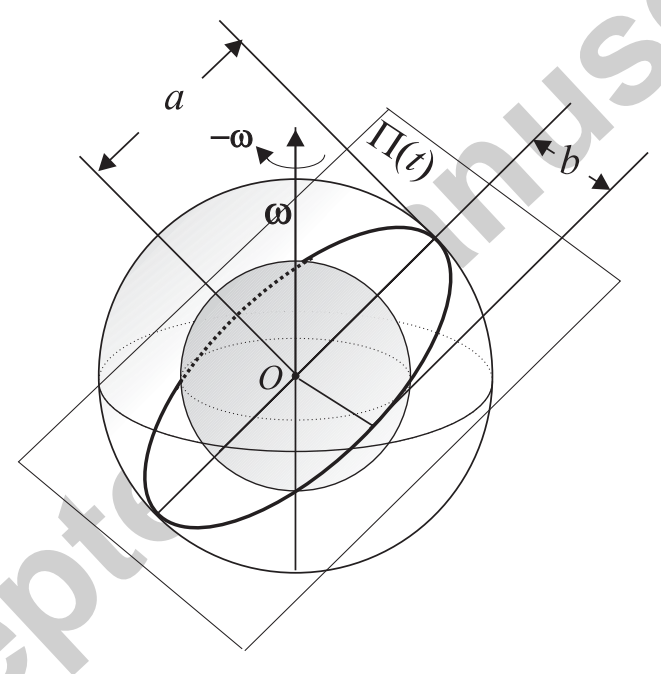

Figure 12: If $\boldsymbol{\Omega}_{0} \neq \mathbf{0}$, the trajectory is a space curve bounded between two concentric spheres.

Two other vectorial conservation laws of the Foucault Pendulum problem follow from the considerations above. Since $\mathbf{a}_{0}$ and $\mathbf{b}_{0}$ are vectorial conservation laws of the initial value problem (4.2), the corresponding conservation laws of the initial value problem (4.1) result to be:

$$
\begin{aligned}
& \mathbf{a}=\mathbf{R}_{-\boldsymbol{\omega}} \mathbf{a}_{0}=\left(\mathbf{R}_{-\boldsymbol{\omega}} \mathbf{r}_{0}\right) \cos \gamma+\left(\mathbf{R}_{-\boldsymbol{\omega}} \mathbf{r}_{0}^{*}\right) \sin \gamma, \\
& \mathbf{b}=\mathbf{R}_{-\boldsymbol{\omega}} \mathbf{b}_{0}=-\left(\mathbf{R}_{-\boldsymbol{\omega}} \mathbf{r}_{0}\right) \sin \gamma+\left(\mathbf{R}_{-\boldsymbol{\omega}} \mathbf{r}_{0}^{*}\right) \cos \gamma
\end{aligned}
$$

This means vectors $\mathbf{a}$ and $\mathbf{b}$ are the results of a rotation with angular velocity $-\boldsymbol{\omega}$ of two constant vectors $\mathbf{a}_{0}$ and $\mathbf{b}_{0}$ defined in eqs (4.11). The law of motion may be now rewritten as:

$$
\mathbf{r}(t)=\left(\mathbf{R}_{-\boldsymbol{\omega}} \mathbf{a}_{0}\right) \cos \left[\omega_{*}\left(t-t_{0}\right)-\gamma\right]+\left(\mathbf{R}_{-\boldsymbol{\omega}} \mathbf{b}_{0}\right) \sin \left[\omega_{*}\left(t-t_{0}\right)-\gamma\right] .
$$

An interesting remark is that from (4.11) the vectorial form of Apollonius Theorems (see [49]) may be deduced by elementary computations:

$$
\mathbf{a}_{0} \times \mathbf{b}_{0}=\mathbf{r}_{0} \times \mathbf{r}_{0}^{*} ; \quad \mathbf{a}_{0}^{2}+\mathbf{b}_{0}^{2}=\mathbf{r}_{0}^{2}+\mathbf{r}_{0}^{* 2}
$$

(vectors $\mathbf{r}_{0}$ and $\mathbf{r}_{0}^{*}$ represent a pair of ellipse conjugate semi-diameters). 
From eqs (4.17) it follows that a tensor variant of Apollonius Theorems (also see [42]) may be given: denoting with " $\otimes$ " the dyadic product of two vectors $\left((\mathbf{u} \otimes \mathbf{v}) \mathbf{x}=(\mathbf{v} \cdot \mathbf{x}) \mathbf{u},(\forall) \mathbf{u}, \mathbf{v}, \mathbf{x} \in \mathbf{V}_{3}^{\mathbb{R}}\right)$, it holds true:

$$
\mathbf{a}_{0} \otimes \mathbf{a}_{0}+\mathbf{b}_{0} \otimes \mathbf{b}_{0}=\mathbf{r}_{0} \otimes \mathbf{r}_{0}+\mathbf{r}_{0}^{*} \otimes \mathbf{r}_{0}^{*}
$$

This result may be reformulated as it follows: if $\mathbf{d}$ and $\mathbf{d}^{*}$ are two arbitrary conjugate semi-diameters of an ellipse, then the tensor:

$$
\mathbf{T}=\mathbf{d} \otimes \mathbf{d}+\mathbf{d}^{*} \otimes \mathbf{d}^{*}
$$

is invariant.

When vector $\boldsymbol{\omega}$ has constant direction, the trajectory is situated inside a spherical segment, as plane $\boldsymbol{\Pi}(t)$ has a precession motion (see Figure 13).

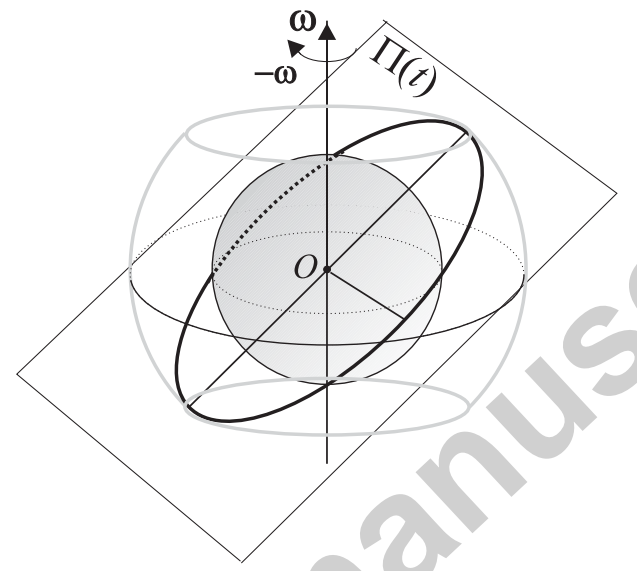

Figure 13: If vector $\boldsymbol{\omega}$ has constant direction, the trajectory is bounded in a spherical segment.

In the particular case when:

$$
\left\{\begin{array}{l}
\left|\mathbf{r}_{0}\right|=\frac{\left|\mathbf{v}_{0}+\omega_{0} \times \mathbf{r}_{0}\right|}{\omega_{*}} \\
\mathbf{r}_{0} \cdot \mathbf{v}_{0}=0
\end{array}\right.
$$

the trajectory in plane $\boldsymbol{\Pi}(t)$ is circular. The trajectory of the Foucault Pendulum results to be a spherical curve.

Generally, the Foucault Pendulum trajectory is a space curve, not necessarily closed. The trajectory is closed if and only if the motion is periodic. This is accomplished when the tensor map $\mathbf{R}_{-\boldsymbol{\omega}}$ is periodic and its main period $T$ satisfies:

$$
\frac{\omega_{*} T}{2 \pi} \in \mathbb{Q}
$$

$\left(\mathbb{Q}\right.$ denotes the set of rational numbers). If vector $\boldsymbol{\omega}$ is constant, then $T=\frac{2 \pi}{\omega}$ and the condition (4.21) becomes:

$$
\frac{\omega_{*}}{\omega} \in \mathbb{Q} .
$$

In a very particular case, the trajectory may be a plane curve. A sufficient condition for that is given in Section 3.3.1: the vector function $\boldsymbol{\omega}$ has constant direction and $\boldsymbol{\omega} \times \boldsymbol{\Omega}_{0}=\mathbf{0}$.

\section{The Plane Trajectory of the Foucault Pendulum}

If $\boldsymbol{\Omega}_{0} \neq \mathbf{0}$, the trajectory may be a plane curve if certain conditions are satisfied. Even is this case, the trajectory may have surprising aspects.

It is a plane curve bounded in an annulus between two concentric circles, one with radius $a$ and the other with radius $b$, where $a$ and $b$ are the magnitudes of vectors $\mathbf{a}$ and $\mathbf{b}$ defined in eqs (4.15). 
The motion is periodic if and only if the tensor map $\mathbf{R}_{-\boldsymbol{\omega}}$ is periodic with the main period $T$ and:

$$
\frac{2 \pi}{\omega_{*} T} \in \mathbb{Q}
$$

In this case the trajectory is a plane closed curve.

When the ratio $\frac{2 \pi}{\omega_{*} T}$ is an irrational number, the trajectory fills densely the annulus where it is bounded on the time interval $\left[t_{0},+\infty\right)$ (see Figure 14$)$.

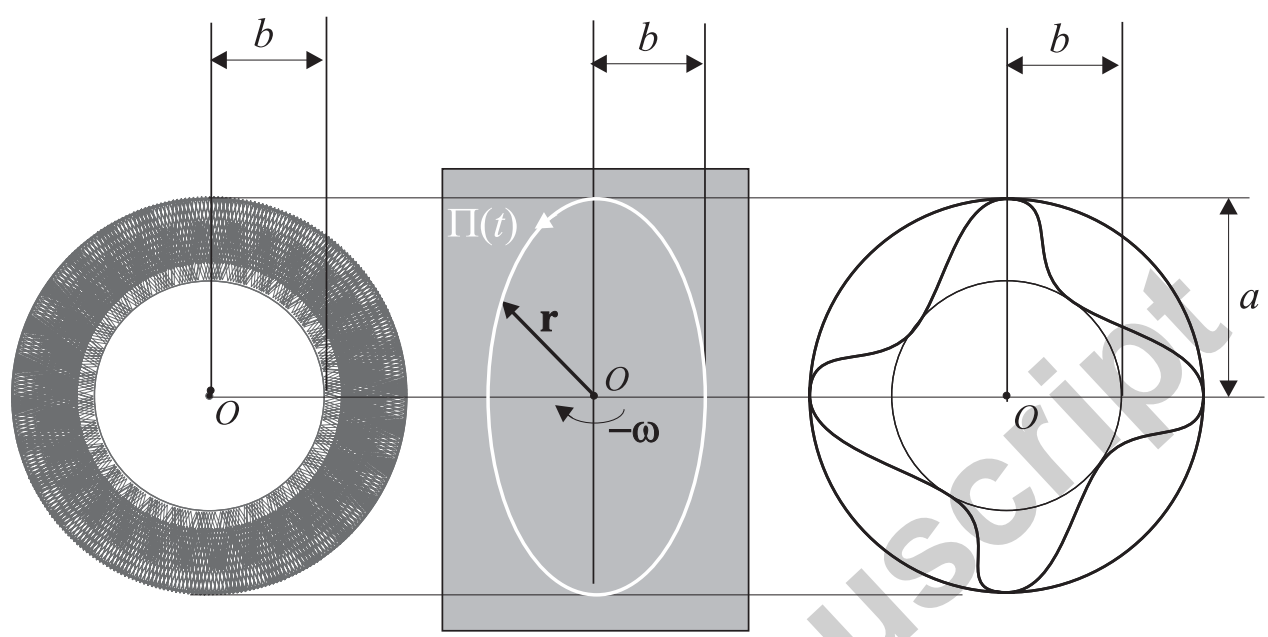

Figure 14: The Foucault Pendulum planar motion: the trajectory is closed or fills densely an annulus.

When $\boldsymbol{\Omega}_{0} \neq \mathbf{0}$, by computing the ratio between the ellipse semiaxes (see Appendix), it follows that:

$$
\begin{aligned}
\frac{a}{b} & =\left[\frac{\mathbf{r}_{0}^{2}+\mathbf{r}_{0}^{* 2}+\sqrt{\left(\mathbf{r}_{0}^{2}-\mathbf{r}_{0}^{* 2}\right)^{2}+4\left(\mathbf{r}_{0} \cdot \mathbf{r}_{0}^{*}\right)^{2}}}{\mathbf{r}_{0}^{2}+\mathbf{r}_{0}^{* 2}-\sqrt{\left(\mathbf{r}_{0}^{2}-\mathbf{r}_{0}^{* 2}\right)^{2}+4\left(\mathbf{r}_{0} \cdot \mathbf{r}_{0}^{*}\right)^{2}}}\right]^{\frac{1}{2}}= \\
& =\frac{\mathbf{r}_{0}^{2}+\mathbf{r}_{0}^{* 2}+\sqrt{\left(\mathbf{r}_{0}^{2}-\mathbf{r}_{0}^{* 2}\right)^{2}+4\left(\mathbf{r}_{0} \cdot \mathbf{r}_{0}^{*}\right)^{2}}}{2\left|\mathbf{r}_{0} \times \mathbf{r}_{0}^{*}\right|},
\end{aligned}
$$

where $\mathbf{r}_{0}^{*}=\frac{\mathbf{v}_{0}+\omega_{0} \times \mathbf{r}_{0}}{\omega_{*}}$.

When $\mathbf{r}_{0} \cdot \mathbf{v}_{0}=0$, then:

$$
\frac{a}{b}=\frac{\max \left(\mathbf{r}_{0}^{2}, \mathbf{r}_{0}^{* 2}\right)}{\left|\mathbf{r}_{0}\right|\left|\mathbf{r}_{0}^{*}\right|}=\left\{\begin{array}{l}
\frac{\left|\mathbf{r}_{0}\right|}{\left|\mathbf{r}_{0}^{*}\right|}, \text { if }\left|\mathbf{r}_{0}\right|>\left|\mathbf{r}_{0}^{*}\right| \\
\frac{\left|\mathbf{r}_{0}^{*}\right|}{\left|\mathbf{r}_{0}\right|}, \text { if }\left|\mathbf{r}_{0}\right|<\left|\mathbf{r}_{0}^{*}\right|
\end{array}\right.
$$

If $\mathbf{v}_{0}=\mathbf{0}, \boldsymbol{\omega}$ is constant and $\boldsymbol{\omega} \cdot \mathbf{r}_{0}=0$, then:

$$
\frac{\left|\mathbf{r}_{0}\right|}{\left|\mathbf{r}_{0}^{*}\right|}=\frac{\omega_{*}}{\omega} .
$$

Making the computations in eq (4.24), we get a generalization of Chevilliet Theorem (see [28], [36]):

$$
\frac{a}{b}=\frac{\max \left(\omega, \omega_{*}\right)}{\min \left(\omega, \omega_{*}\right)}
$$

(the ratio between the elliptic main period and the precession main period equals the ratio between the ellipse semiaxes). 


\subsubsection{Zero Generalized Angular Momentum: $\Omega=0$}

In this particular case, the harmonic oscillation described by the initial value problem (4.2) is rectilinear. This case occurs if and only if:

$$
\mathbf{r}_{0} \times\left(\mathbf{v}_{0}+\boldsymbol{\omega}_{0} \times \mathbf{r}_{0}\right)=\mathbf{0} .
$$

The motion may be decomposed into:

1. a harmonic rectilinear oscillation;

2. a rotation with instantaneous angular velocity $-\boldsymbol{\omega}$ around a fixed point of the straight line where the harmonic oscillation takes place. This rotation is an arbitrary precession if $\boldsymbol{\omega}$ has constant direction or a regular precession if $\boldsymbol{\omega}$ is constant.

\section{The case $\mathbf{r}_{0} \neq 0$}

The pulsation of the harmonic oscillation is $\omega_{*}$. Its amplitude is:

$$
A=\sqrt{\mathbf{r}_{0}^{2}+\left(\frac{\mathbf{r}_{0} \cdot \mathbf{v}_{0}}{\omega_{*} r_{0}}\right)^{2}} .
$$

The law of motion may be written as:

$$
\mathbf{r}(t)=\sqrt{\mathbf{r}_{0}^{2}+\left(\frac{\mathbf{r}_{0} \cdot \mathbf{v}_{0}}{\omega_{*} r_{0}}\right)^{2}} \sin \left[\omega_{*}\left(t-t_{0}\right)+\varphi\right] \mathbf{R}_{-\omega} \frac{\mathbf{r}_{0}}{r_{0}},
$$

where $\varphi \in[0,2 \pi)$ is defined by (see Appendix):

$$
\sin \varphi=\frac{r_{0}}{\sqrt{\mathbf{r}_{0}^{2}+\left(\frac{\mathbf{r}_{0} \cdot \mathbf{v}_{0}}{\omega_{*} r_{0}}\right)^{2}}}, \quad \cos \varphi=\frac{\frac{\mathbf{r}_{0} \cdot \mathbf{v}_{0}}{\omega_{*} r_{0}}}{\sqrt{\mathbf{r}_{0}^{2}+\left(\frac{\mathbf{r}_{0} \cdot \mathbf{v}_{0}}{\omega_{*} r_{0}}\right)^{2}}}
$$

The case $\mathbf{r}_{0}=0$

The law of motion is:

$$
\mathbf{r}(t)=\frac{\mathbf{R}_{-\omega} \mathbf{v}_{0}}{\omega_{*}} \sin \omega_{*}\left(t-t_{0}\right) \text {. }
$$

The harmonic oscillation has the pulsation $\omega_{*}$ and the amplitude:

$$
A=\frac{\left|\mathbf{v}_{0}\right|}{\omega_{*}} .
$$

\section{The space trajectory}

This is the most general case. The trajectory is a curve situated on a conical surface. If vector $\boldsymbol{\omega}$ has constant direction, the trajectory is situated on the lateral surface of a right circular cone. If vector $\boldsymbol{\omega}$ is constant, the trajectory may or may not be closed. The trajectory is a closed curve if and only if the ratio $\frac{\omega}{\omega_{*}}$ is a rational number (see Figure $15)$.

\section{The plane trajectory}

When vector $\boldsymbol{\omega}$ has a fixed direction and $\mathbf{r}_{0} \cdot \boldsymbol{\omega}=0$, the trajectory is a plane curve, as it results from (4.27) and (4.29). The trajectory is bounded inside a circle with the radius:

$$
R=A=\sqrt{\mathbf{r}_{0}^{2}+\left(\frac{\mathbf{r}_{0} \cdot \mathbf{v}_{0}}{\omega_{*} r_{0}}\right)^{2}} .
$$



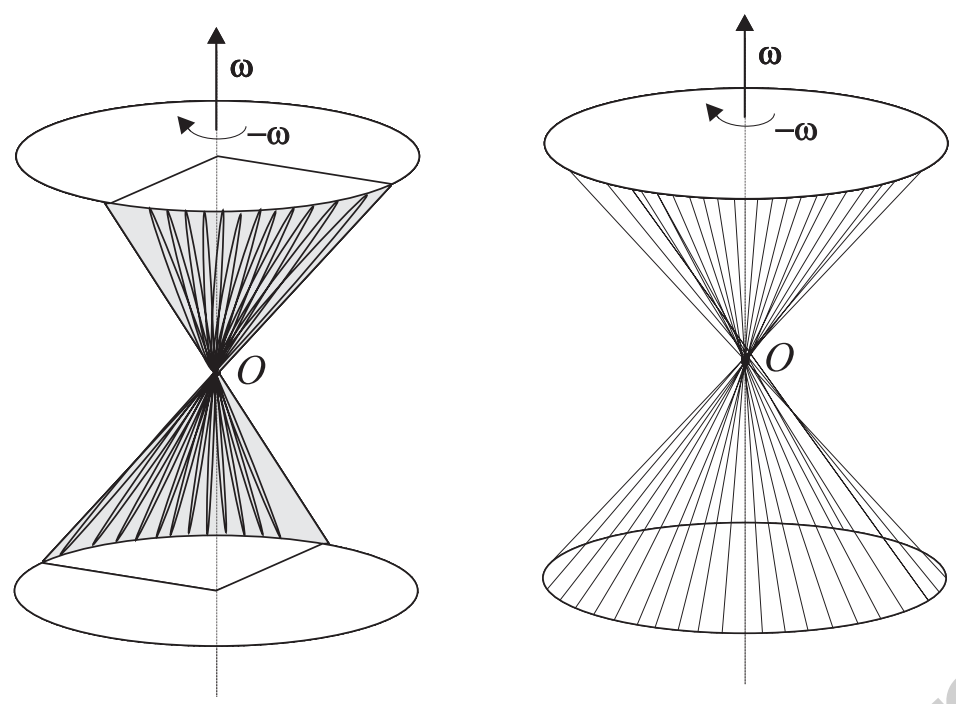

Figure 15: If $\boldsymbol{\Omega}_{0}=\mathbf{0}$ : Left: $\boldsymbol{\omega}$ has constant direction; Right: $\boldsymbol{\omega}$ is constant; the trajectory is situated on the lateral surface of a right circular cone.
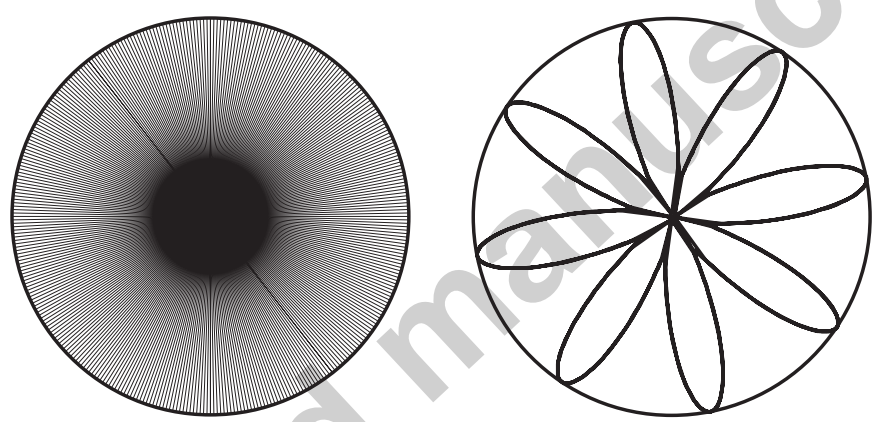

Figure 16: The planar trajectory when $\boldsymbol{\Omega}=\mathbf{0}$. Left: it fills densely a circle. $\mathbf{R i g h t :}$ it is a closed curve.

If $\frac{\omega}{\omega_{*}}$ is a rational number, the trajectory is a closed curve. If $\frac{\omega}{\omega_{*}}$ is an irrational number, the trajectory fills densely a circle (see Figure 16).

The rectilinear trajectory

The trajectory may also be rectilinear, a harmonic oscillation on a straight line. In the sequel, a necessary and sufficient condition for the trajectory to be rectilinear when $\boldsymbol{\Omega}_{0}=\mathbf{0}$ is given.

When $\boldsymbol{\Omega}_{0}=\mathbf{0}$, the trajectory is rectilinear if and only if the following conditions are satisfied:

1. Vector $\boldsymbol{\omega}$ has constant direction;

2. $\boldsymbol{\omega} \times \mathbf{r}_{0}=\mathbf{0}$.

Proof. " $\Rightarrow$ " Supposing the trajectory is rectilinear, from eq (4.29) it follows that $\mathbf{R}_{-\omega} \mathbf{r}_{0}$ is constant. Then:

$$
\frac{d}{d t}\left(\mathbf{R}_{-\omega} \mathbf{r}_{0}\right)=\mathbf{0} \Rightarrow \boldsymbol{\omega} \times \mathbf{R}_{-\omega} \mathbf{r}_{0}=\mathbf{0}
$$

so vector $\boldsymbol{\omega}$ has a constant direction, that of $\mathbf{R}_{-\boldsymbol{\omega}} \mathbf{r}_{0}$. It follows that $\mathbf{R}_{-\boldsymbol{\omega}} \boldsymbol{\omega}=\boldsymbol{\omega}$. By replacing in (4.34), it results $\boldsymbol{\omega} \times \mathbf{r}_{0}=\mathbf{0}$.

$" \Leftarrow$ " If $\boldsymbol{\omega}$ has constant direction and $\boldsymbol{\omega} \times \mathbf{r}_{0}=\mathbf{0}$, then $\mathbf{R}_{-\boldsymbol{\omega}}\left(\boldsymbol{\omega} \times \mathbf{r}_{0}\right)=\mathbf{0}$, so $\boldsymbol{\omega} \times \mathbf{R}_{-\boldsymbol{\omega}} \mathbf{r}_{0}=\mathbf{0}$. The continuous vectorial map $\mathbf{R}_{-\boldsymbol{\omega}} \mathbf{r}_{0}$ has constant magnitude and constant direction, so it is constant. From eq (4.29) the motion results to be rectilinear.

When $\boldsymbol{\Omega}_{0}=\mathbf{0}$, the motion is rectilinear if the rotation with angular velocity $\boldsymbol{- \boldsymbol { \omega }}$ has no effect on the straight line where the rectilinear harmonic oscillation takes place. This situation occurs when vector $\boldsymbol{\omega}$ has the fixed direction of $\mathbf{r}_{0}$. 


\section{The Reduced Foucault Pendulum Problem}

Traditionally, in most Theoretical Mechanics textbooks, the initial value problem that describes the Foucault Pendulum is considered to be (see [29]-[32]):

$$
\left\{\begin{array}{l}
\ddot{\mathbf{r}}+2 \boldsymbol{\omega} \times \dot{\mathbf{r}}+\omega_{*}^{2} \mathbf{r}=\mathbf{0}, \omega_{*}>0 \\
\mathbf{r}\left(t_{0}\right)=\mathbf{r}_{0} ; \dot{\mathbf{r}}\left(t_{0}\right)=\mathbf{v}_{0}
\end{array}\right.
$$

with $\boldsymbol{\omega}$ a constant vector, $\boldsymbol{\omega}=\boldsymbol{\omega}_{\text {Earth }} \sin \psi$, where $\psi$ is the latitude of the place where the experiment is performed. Only the Coriolis acceleration is taken into account in eq (5.1). The term $\boldsymbol{\omega} \times(\boldsymbol{\omega} \times \mathbf{r})$ is neglected.

Solutions to this initial value problem are offered only in the planar case, using polar coordinates (see [28]), Cartesian coordinates (see [30]) or complex numbers (see [29]). Using the tensor instrument introduced in Section 2, we will also obtain an explicit solution to this initial value problem.

Since vector $\boldsymbol{\omega}$ is constant, we are allowed to decompose the linear initial value problem (5.1) into two initial value problems, one whose the solution has the direction of vector $\boldsymbol{\omega}$ and another problem, whose solution is orthogonal to $\boldsymbol{\omega}$. In fact, the solution to the initial value problem (5.1) is:

$$
\mathbf{r}=\mathbf{r}_{\|}+\mathbf{r}_{\perp}
$$

where $\mathbf{r}_{\|}$is the solution to the initial value problem:

$$
\left\{\begin{array}{l}
\ddot{\mathbf{r}}+\omega_{*}^{2} \mathbf{r}=\mathbf{0} \\
\mathbf{r}\left(t_{0}\right)=\frac{\boldsymbol{\omega} \cdot \mathbf{r}_{0}}{\omega^{2}} \boldsymbol{\omega} ; \dot{\mathbf{r}}\left(t_{0}\right)=\frac{\boldsymbol{\omega} \cdot \mathbf{v}_{0}}{\omega^{2}} \boldsymbol{\omega}
\end{array}\right.
$$

and $\mathbf{r}_{\perp}$ to the initial value problem:

$$
\left\{\begin{array}{l}
\ddot{\mathbf{r}}+2 \boldsymbol{\omega} \times \dot{\mathbf{r}}+\omega_{*}^{2} \mathbf{r}=\mathbf{0} \\
\mathbf{r}\left(t_{0}\right)=\frac{\boldsymbol{\omega} \times\left(\mathbf{r}_{0} \times \boldsymbol{\omega}\right)}{\omega^{2}} ; \dot{\mathbf{r}}\left(t_{0}\right)=\frac{\boldsymbol{\omega} \times\left(\mathbf{v}_{0} \times \boldsymbol{\omega}\right)}{\omega^{2}}
\end{array}\right.
$$

The solution to the initial value problem (5.3) is obtained as:

$$
\mathbf{r}_{\|}=\left\{\frac{\boldsymbol{\omega} \cdot \mathbf{r}_{0}}{\omega^{2}} \cos \left[\omega_{*}\left(t-t_{0}\right)\right]+\frac{\boldsymbol{\omega} \cdot \mathbf{v}_{0}}{\omega_{*} \omega^{2}} \sin \left[\omega_{*}\left(t-t_{0}\right)\right]\right\} \boldsymbol{\omega} .
$$

By denoting $\omega^{*}=\sqrt{\omega^{2}+\omega_{*}^{2}}$, it follows that the solution to the initial value problem (5.4) is:

$$
\mathbf{r}_{\perp}=-\frac{1}{\omega^{2}} \mathbf{R}_{-\boldsymbol{\omega}}\left(\widetilde{\boldsymbol{\omega}}^{2} \mathbf{r}_{0}\right) \cos \left[\omega^{*}\left(t-t_{0}\right)\right]+\frac{1}{\omega^{*}} \mathbf{R}_{-\boldsymbol{\omega}}\left(\widetilde{\boldsymbol{\omega}} \mathbf{r}_{0}-\frac{\widetilde{\boldsymbol{\omega}}^{2}}{\omega^{2}} \mathbf{v}_{0}\right) \sin \left[\omega^{*}\left(t-t_{0}\right)\right]
$$

Making all computations in (5.6), it follows:

$$
\begin{aligned}
\mathbf{r}_{\perp}(t)= & -\left(\frac{\sin \left[\omega\left(t-t_{0}\right)\right]}{\omega} \widetilde{\boldsymbol{\omega}} \mathbf{r}_{0}+\frac{\cos \left[\omega\left(t-t_{0}\right)\right]}{\omega^{2}} \widetilde{\boldsymbol{\omega}}^{2} \mathbf{r}_{0}\right) \cos \left[\omega^{*}\left(t-t_{0}\right)\right]+ \\
& +\frac{1}{\omega^{*}}\left(-\frac{\sin \left[\omega\left(t-t_{0}\right)\right]}{\omega} \widetilde{\boldsymbol{\omega}}^{2} \mathbf{r}_{0}+\cos \left[\omega\left(t-t_{0}\right)\right] \widetilde{\boldsymbol{\omega}} \mathbf{r}_{0}-\frac{\sin \left[\omega\left(t-t_{0}\right)\right]}{\omega} \widetilde{\boldsymbol{\omega}} \mathbf{v}_{0}-\right. \\
& \left.-\frac{\cos \left[\omega\left(t-t_{0}\right)\right]}{\omega^{2}} \widetilde{\boldsymbol{\omega}}^{2} \mathbf{v}_{0}\right) \sin \left[\omega^{*}\left(t-t_{0}\right)\right] .
\end{aligned}
$$

It results that the explicit solution to the initial value problem (5.1) is expressed in eq (5.2), where $\mathbf{r}_{\|}$is given in eq (5.5) and $\mathbf{r}_{\perp}$ in (5.7).

We obtain that the solution to the initial value problem (5.1) is:

$$
\mathbf{r}(t)=\frac{\boldsymbol{\omega} \cdot \mathbf{\Phi}_{*}}{\omega^{2}} \boldsymbol{\omega}-\sin \left[\omega\left(t-t_{0}\right)\right] \frac{\boldsymbol{\omega} \times \boldsymbol{\Phi}^{*}}{\omega}-\cos \left[\omega\left(t-t_{0}\right)\right] \frac{\boldsymbol{\omega} \times\left(\boldsymbol{\omega} \times \boldsymbol{\Phi}^{*}\right)}{\omega^{2}},
$$


where:

$$
\left\{\begin{array}{l}
\boldsymbol{\Phi}_{*}=\mathbf{r}_{0} \cos \left[\omega_{*}\left(t-t_{0}\right)\right]+\frac{\mathbf{v}_{0}+\boldsymbol{\omega} \times \mathbf{r}_{0}}{\omega_{*}} \sin \left[\omega_{*}\left(t-t_{0}\right)\right] \\
\boldsymbol{\Phi}^{*}=\mathbf{r}_{0} \cos \left[\omega^{*}\left(t-t_{0}\right)\right]+\frac{\mathbf{v}_{0}+\boldsymbol{\omega} \times \mathbf{r}_{0}}{\omega^{*}} \sin \left[\omega^{*}\left(t-t_{0}\right)\right] \\
\omega^{*}=\sqrt{\omega^{2}+\omega_{*}^{2}}
\end{array}\right.
$$

One may remark that by adding and subtracting $\frac{1}{\omega^{2}}\left(\boldsymbol{\omega} \cdot \boldsymbol{\Phi}^{*}\right) \boldsymbol{\omega}$, eq (5.8) becomes:

$$
\mathbf{r}(t)=\frac{\boldsymbol{\omega} \cdot\left(\boldsymbol{\Phi}_{*}-\boldsymbol{\Phi}^{*}\right)}{\omega^{2}} \boldsymbol{\omega}+\mathbf{R}_{-\boldsymbol{\omega}} \boldsymbol{\Phi}^{*}
$$

The motion described by eq (5.10) is a composition among:

1. a composition of two asynchronous rectilinear oscillations with pulsations $\omega_{*}$ and $\omega^{*}=\sqrt{\omega^{2}+\omega_{*}^{2}}$ along a straight line that has the direction of vector $\boldsymbol{\omega}$;

2. an elliptic oscillation with pulsation $\omega^{*}=\sqrt{\omega^{2}+\omega_{*}^{2}}$ in a plane that is orthogonal to vector $\boldsymbol{\omega}$;

3. a precession with angular velocity $-\boldsymbol{\omega}$ of the plane where the elliptic oscillation takes place.

If $\mathbf{r}_{0} \cdot \boldsymbol{\omega}=0, \mathbf{v}_{0} \cdot \boldsymbol{\omega}=0$, eq $(5.10)$ becomes:

$$
\mathbf{r}(t)=\mathbf{R}_{-\boldsymbol{\omega}} \Phi^{*}
$$

The motion is planar and it may be decomposed into:

(i) an elliptic oscillation in a plane that is orthogonal on vector $\boldsymbol{\omega}$;

(ii) a precession with angular velocity $-\boldsymbol{\omega}$ of the plane where the elliptic oscillation takes place.

If $\mathbf{r}_{0} \cdot \boldsymbol{\omega}=0, \mathbf{v}_{0}=\mathbf{0}$, eq (5.10) becomes:

$$
\begin{aligned}
\mathbf{r}(t) & =\mathbf{R}_{-\boldsymbol{\omega}} \boldsymbol{\Phi}^{*}=-\sin \left[\omega\left(t-t_{0}\right)\right] \frac{\boldsymbol{\omega} \times \boldsymbol{\Phi}^{*}}{\omega}-\cos \left[\omega\left(t-t_{0}\right)\right] \frac{\boldsymbol{\omega} \times\left(\boldsymbol{\omega} \times \boldsymbol{\Phi}^{*}\right)}{\omega^{2}}, \\
\boldsymbol{\Phi}^{*} & =\mathbf{r}_{0} \cos \left[\omega^{*}\left(t-t_{0}\right)\right]+\frac{\boldsymbol{\omega} \times \mathbf{r}_{0}}{\omega^{*}} \sin \left[\omega^{*}\left(t-t_{0}\right)\right] .
\end{aligned}
$$

Making all computations in eq (5.12), it results:

$$
\mathbf{r}(t)=\cos \left[\omega\left(t-t_{0}\right)\right] \boldsymbol{\Phi}^{*}-\sin \left[\omega\left(t-t_{0}\right)\right] \frac{\boldsymbol{\omega} \times \boldsymbol{\Phi}^{*}}{\omega},
$$

and further:

$$
\begin{aligned}
\mathbf{r}(t)= & \left\{\cos \left[\omega\left(t-t_{0}\right)\right] \cos \left[\omega^{*}\left(t-t_{0}\right)\right]-\frac{\omega}{\omega^{*}} \sin \left[\omega\left(t-t_{0}\right)\right] \sin \left[\omega^{*}\left(t-t_{0}\right)\right]\right\} \mathbf{r}_{0}+ \\
& +\left\{\frac{\omega}{\omega^{*}} \sin \left[\omega^{*}\left(t-t_{0}\right)\right] \cos \left[\omega\left(t-t_{0}\right)\right]-\sin \left[\omega\left(t-t_{0}\right)\right] \cos \left[\omega^{*}\left(t-t_{0}\right)\right]\right\} \frac{\boldsymbol{\omega} \times \mathbf{r}_{0}}{\omega} .
\end{aligned}
$$

Eq (5.12) represents the vectorial form of the traditional solution to the Foucault Pendulum problem given in most Theoretical Mechanics textbooks. Denoting:

$$
\mathbf{i}=\frac{\mathbf{r}_{0}}{r_{0}} ; \quad \mathbf{j}=\frac{\boldsymbol{\omega} \times \mathbf{r}_{0}}{\omega r_{0}}
$$

the parametric Cartesian form of eq (5.14) is:

$$
\left\{\begin{array}{l}
x=\left\{\cos \left[\omega\left(t-t_{0}\right)\right] \cos \left[\omega^{*}\left(t-t_{0}\right)\right]-\frac{\omega}{\omega^{*}} \sin \left[\omega\left(t-t_{0}\right)\right] \sin \left[\omega^{*}\left(t-t_{0}\right)\right]\right\} r_{0}, \\
y=\left\{\frac{\omega}{\omega^{*}} \sin \left[\omega^{*}\left(t-t_{0}\right)\right] \cos \left[\omega\left(t-t_{0}\right)\right]-\sin \left[\omega\left(t-t_{0}\right)\right] \cos \left[\omega^{*}\left(t-t_{0}\right)\right]\right\} r_{0} .
\end{array}\right.
$$




\section{The Harmonic Oscillator in a Magnetic Field}

The reduced initial value problem (5.1) also models the motion of a harmonic oscillator in a uniform magnetic field. Indeed, considering $P(m, q)$ a particle with mass $m$ and electric charge $q$, its motion is described by the initial value problem:

$$
\left\{\begin{array}{l}
m \ddot{\mathbf{r}}=q(\dot{\mathbf{r}} \times \mathbf{B})-k \mathbf{r} \\
\mathbf{r}(0)=\mathbf{r}_{0} ; \dot{\mathbf{r}}(0)=\mathbf{v}_{0}
\end{array}\right.
$$

where $\mathbf{B}$ is the magnetic field and $k>0$ the elastic constant. This problem is exactly the same as (5.1). By denoting:

$$
\boldsymbol{\omega}=\frac{q \mathbf{B}}{2 m} ; \quad \omega_{*}=\sqrt{\frac{k}{m}},
$$

eq (5.17) becomes the initial value problem (5.1) with the solution given in eq (5.8).

\section{The Driven Foucault Pendulum}

This section offers, for the first time (by the knowledge of the authors), a closed form solution to the driven Foucault Pendulum problem. Both the general and the reduced cases are treated.

\subsection{The Exact Solution to the Driven Foucault Pendulum Problem}

Suppose that on the particle whose motion is studied an additional force is applied. It has the expression:

$$
\mathbf{F}=m \mathbf{f}(t) .
$$

By using the tensor instrument introduced in Section 2, an exact solution to the driven Foucault Pendulum problem is offered. The problem is modeled by the initial value problem:

$$
\left\{\begin{array}{l}
\ddot{\mathbf{r}}+2 \boldsymbol{\omega} \times \dot{\mathbf{r}}+\boldsymbol{\omega} \times(\boldsymbol{\omega} \times \mathbf{r})+\dot{\boldsymbol{\omega}} \times \mathbf{r}+\omega_{*}^{2} \mathbf{r}=\mathbf{f}(t) \\
\mathbf{r}\left(t_{0}\right)=\mathbf{r}_{0} ; \dot{\mathbf{r}}\left(t_{0}\right)=\mathbf{v}_{0}
\end{array}\right.
$$

where $\mathbf{f}: \mathbb{R} \rightarrow \mathbf{V}_{3}$ is a continuous vectorial map.

We may state that the solution to the initial value problem (6.2) is:

$$
\mathbf{r}(t)=\mathbf{R}_{-\boldsymbol{\omega}}\left\{\mathbf{r}_{0} \cos \left[\omega_{*}\left(t-t_{0}\right)\right]+\frac{\mathbf{v}_{0}+\boldsymbol{\omega}_{0} \times \mathbf{r}_{0}}{\omega_{*}} \sin \left[\omega_{*}\left(t-t_{0}\right)\right]+\frac{1}{\omega_{*}}\left(\sin \omega_{*} t\right) *\left(\mathbf{R}_{-\boldsymbol{\omega}}^{T} \mathbf{f}\right)\right\},
$$

where "*" denotes the convolution product of two functions:

$$
g * h \stackrel{\text { def }}{=} \int_{t_{0}}^{t} g(t-\tau) h(\tau) d \tau .
$$

Proof. By applying the tensor operator $\mathbf{F}_{\boldsymbol{\omega}}$, the initial value problem (6.2) becomes:

$$
\left\{\begin{array}{l}
\frac{d^{2}}{d t^{2}}\left(\mathbf{F}_{\boldsymbol{\omega}} \mathbf{r}\right)+\omega_{*}^{2}\left(\mathbf{F}_{\boldsymbol{\omega}} \mathbf{r}\right)=\mathbf{F}_{\boldsymbol{\omega}} \mathbf{f}, \\
\left(\mathbf{F}_{\boldsymbol{\omega}} \mathbf{r}\right)\left(t_{0}\right)=\mathbf{r}_{0} ;\left[\frac{d}{d t}\left(\mathbf{F}_{\boldsymbol{\omega}} \mathbf{r}\right)\right]\left(t_{0}\right)=\mathbf{v}_{0}+\boldsymbol{\omega}_{0} \times \mathbf{r}_{0} .
\end{array}\right.
$$

From Section 4 it results that the solution to the homogenous initial value problem attached to eq (6.5) is:

$$
\left(\mathbf{F}_{\boldsymbol{\omega}} \mathbf{r}\right)_{0}(t)=\mathbf{r}_{0} \cos \left[\omega_{*}\left(t-t_{0}\right)\right]+\frac{\mathbf{v}_{0}+\boldsymbol{\omega}_{0} \times \mathbf{r}_{0}}{\omega_{*}} \sin \left[\omega_{*}\left(t-t_{0}\right)\right] .
$$

Applying the method of variation of coefficients, we seek for a solution for the initial value problem (6.5) of the form:

$$
\left(\mathbf{F}_{\boldsymbol{\omega}} \mathbf{r}\right)(t)=\left[\mathbf{r}_{0}+\mathbf{K}_{1}(t)\right] \cos \left[\omega_{*}\left(t-t_{0}\right)\right]+\left[\frac{\mathbf{v}_{0}+\omega_{0} \times \mathbf{r}_{0}}{\omega_{*}}+\mathbf{K}_{2}(t)\right] \sin \left[\omega_{*}\left(t-t_{0}\right)\right],
$$


where the vectorial maps $\mathbf{K}_{1}$ and $\mathbf{K}_{2}$ are solutions to the linear ODE system:

$$
\left\{\begin{array}{c}
\dot{\mathbf{K}}_{1} \cos \left[\omega_{*}\left(t-t_{0}\right)\right]+\dot{\mathbf{K}}_{2} \sin \left[\omega_{*}\left(t-t_{0}\right)\right]=\mathbf{0}, \\
-\dot{\mathbf{K}}_{1} \sin \left[\omega_{*}\left(t-t_{0}\right)\right]+\dot{\mathbf{K}}_{2} \cos \left[\omega_{*}\left(t-t_{0}\right)\right]=\frac{\mathbf{F}_{\boldsymbol{\omega}} \mathbf{f}}{\omega_{*}} .
\end{array}\right.
$$

After computations, eq (6.7) becomes:

$$
\left(\mathbf{F}_{\boldsymbol{\omega}} \mathbf{r}\right)(t)=\mathbf{r}_{0} \cos \left[\omega_{*}\left(t-t_{0}\right)\right]+\frac{\mathbf{v}_{0}+\boldsymbol{\omega}_{0} \times \mathbf{r}_{0}}{\omega_{*}} \sin \left[\omega_{*}\left(t-t_{0}\right)\right]+\frac{1}{\omega_{*}} \int_{t_{0}}^{t} \sin \left[\omega_{*}(t-\tau)\right]\left(\mathbf{F}_{\boldsymbol{\omega}} \mathbf{f}\right) d \tau .
$$

Applying the tensor map $\mathbf{R}_{-\boldsymbol{\omega}}$ to eq (6.9) and taking eq (6.4) into account, it follows that the conclusion of the theorem is proved. The proof is finalized.

One may remark that if vector $\omega$ has constant direction, $\boldsymbol{\omega}=\omega(t) \mathbf{u}$, with $\omega: \mathbb{R}_{+} \rightarrow \mathbb{R}$ and $\mathbf{u}$ its constant unit vector, then $\mathbf{R}_{-\boldsymbol{\omega}}^{T}=\left(\mathbf{R}_{-\boldsymbol{\omega}}\right)^{-1}=\mathbf{R}_{\boldsymbol{\omega}}$ and eq (6.3) becomes:

$$
\mathbf{r}(t)=\mathbf{R}_{-\boldsymbol{\omega}}\left\{\mathbf{r}_{0} \cos \left[\omega_{*}\left(t-t_{0}\right)\right]+\frac{\mathbf{v}_{0}+\boldsymbol{\omega}_{0} \times \mathbf{r}_{0}}{\omega_{*}} \sin \left[\omega_{*}\left(t-t_{0}\right)\right]+\frac{1}{\omega_{*}}\left(\sin \omega_{*} t\right) *\left(\mathbf{R}_{\boldsymbol{\omega}} \mathbf{f}\right)\right\},
$$

where:

$$
\left\{\begin{array}{l}
\mathbf{R}_{\boldsymbol{\omega}(t)}=\mathbf{I}_{3}+\frac{\sin \varphi(t)}{\omega} \widetilde{\boldsymbol{\omega}}+\frac{1-\cos \varphi(t)}{\omega^{2}} \widetilde{\boldsymbol{\omega}}^{2}, \\
\varphi(t)=\int_{t_{0}}^{t} \omega(\xi) d \xi .
\end{array}\right.
$$

The Foucault Pendulum Motion with Respect to an Arbitrary Non-inertial Reference Frame

In the case of an arbitrary motion of the non-inertial reference frame in the driven Foucault Pendulum problem, $\mathbf{a}(t)$ denotes the acceleration of the origin of the non-inertial reference frame. The initial value problem (6.2) models the motion with respect to this reference frame by replacing $\mathbf{f}(t)=-\mathbf{a}(t)$. Eq (6.3) gives the exact vectorial closed form solution to the driven Foucault Pendulum problem with respect to an arbitrary non-inertial reference frame, that is:

$$
\mathbf{r}(t)=\mathbf{R}_{-\boldsymbol{\omega}}\left\{\mathbf{r}_{0} \cos \left[\omega_{*}\left(t-t_{0}\right)\right]+\frac{\mathbf{v}_{0}+\boldsymbol{\omega}_{0} \times \mathbf{r}_{0}}{\omega_{*}} \sin \left[\omega_{*}\left(t-t_{0}\right)\right]-\frac{1}{\omega_{*}}\left(\sin \omega_{*} t\right) *\left(\mathbf{R}_{-\boldsymbol{\omega}}^{T} \mathbf{a}\right)\right\}
$$

\subsection{The Exact Solution to the Driven Reduced Foucault Pendulum Problem}

The tensor instrument introduced in Section 2 allows to give the solution to the driven reduced Foucault Pendulum problem, that is described by the initial value problem:

$$
\left\{\begin{array}{l}
\ddot{\mathbf{r}}+2 \boldsymbol{\omega} \times \dot{\mathbf{r}}+\omega_{*}^{2} \mathbf{r}=\mathbf{f}(\mathbf{t}), \omega_{*}>0 \\
\mathbf{r}\left(t_{0}\right)=\mathbf{r}_{0}, \dot{\mathbf{r}}\left(t_{0}\right)=\mathbf{v}_{0}
\end{array}\right.
$$

where $\mathbf{f}: \mathbb{R} \rightarrow \mathbf{V}_{3}$ is a continuous vectorial map.

The same procedure as in Section 5 is used: we will split the initial value problem (6.13) into two distinct initial value problems. By adding the solutions to these initial value problems, the solution to the initial value problem (6.13) is obtained.

We consider $\mathbf{r}=\mathbf{r}_{\|}+\mathbf{r}_{\perp}$, where $\mathbf{r}_{\|}$is the solution to the initial value problem:

$$
\left\{\begin{array}{l}
\ddot{\mathbf{r}}+\omega_{*}^{2} \mathbf{r}=\frac{\boldsymbol{\omega} \cdot \mathbf{f}}{\omega^{2}} \boldsymbol{\omega}, \\
\mathbf{r}\left(t_{0}\right)=\frac{\boldsymbol{\omega} \cdot \mathbf{r}_{0}}{\omega^{2}} \boldsymbol{\omega} ; \dot{\mathbf{r}}\left(t_{0}\right)=\frac{\boldsymbol{\omega} \cdot \mathbf{v}_{0}}{\omega^{2}} \boldsymbol{\omega}
\end{array}\right.
$$


and $\mathbf{r}_{\perp}$ to the initial value problem:

$$
\left\{\begin{array}{l}
\ddot{\mathbf{r}}+2 \boldsymbol{\omega} \times \dot{\mathbf{r}}+\omega_{*}^{2} \mathbf{r}=\frac{\boldsymbol{\omega} \times(\mathbf{f} \times \boldsymbol{\omega})}{\omega^{2}} \\
\mathbf{r}\left(t_{0}\right)=\frac{\boldsymbol{\omega} \times\left(\mathbf{r}_{0} \times \boldsymbol{\omega}\right)}{\omega^{2}} ; \dot{\mathbf{r}}\left(t_{0}\right)=\frac{\boldsymbol{\omega} \times\left(\mathbf{v}_{0} \times \boldsymbol{\omega}\right)}{\omega^{2}}
\end{array}\right.
$$

The solution to the initial value problem (6.13) is:

$$
\begin{aligned}
\mathbf{r}= & \frac{\boldsymbol{\omega} \cdot \Phi_{*}}{\omega^{2}} \boldsymbol{\omega}-\sin \left[\omega\left(t-t_{0}\right)\right] \frac{\boldsymbol{\omega} \times \Phi^{*}}{\omega}-\cos \left[\omega\left(t-t_{0}\right)\right] \frac{\boldsymbol{\omega} \times\left(\boldsymbol{\omega} \times \Phi^{*}\right)}{\omega^{2}}+ \\
& \frac{\left(\sin \omega_{*} t * \mathbf{f}\right) \cdot \boldsymbol{\omega}}{\omega_{*} \omega^{2}} \boldsymbol{\omega}+\mathbf{R}_{-\boldsymbol{\omega}}\left[\frac{\sin \omega^{*} t}{\omega^{*}} * \frac{\boldsymbol{\omega} \times\left(\mathbf{R}_{\boldsymbol{\omega}} \mathbf{f} \times \boldsymbol{\omega}\right)}{\omega^{2}}\right]
\end{aligned}
$$

where $\omega^{*}=\sqrt{\omega^{2}+\omega_{*}^{2}}$ and vectors $\boldsymbol{\Phi}_{*}$ and $\boldsymbol{\Phi}^{*}$ are defined by eqs (5.9).

Eq (6.16) offers the exact solution to the reduced form of the driven Foucault Pendulum problem. Since vector $\boldsymbol{\omega}$ is constant, $\mathbf{F}_{\boldsymbol{\omega}}=\mathbf{R}_{\boldsymbol{\omega}}$.

With vectors $\boldsymbol{\Phi}_{*}$ and $\boldsymbol{\Phi}^{*}$ defined by eqs (5.9), eq (6.16) may be rewritten:

$$
\begin{aligned}
\mathbf{r}= & \frac{\boldsymbol{\omega} \cdot\left(\Phi_{*}-\Phi^{*}\right)}{\omega^{2}} \boldsymbol{\omega}+\mathbf{R}_{-\boldsymbol{\omega}} \Phi^{*}+ \\
& +\frac{\left(\sin \omega_{*} t * \mathbf{f}\right) \cdot \boldsymbol{\omega}}{\omega_{*} \omega^{2}} \boldsymbol{\omega}+\frac{1}{\omega^{2}} \mathbf{R}_{-\boldsymbol{\omega}}\left\{\frac{\sin \omega^{*} t}{\omega^{*}} * \mathbf{R}_{\boldsymbol{\omega}}[\boldsymbol{\omega} \times(\mathbf{f} \times \boldsymbol{\omega})]\right\},
\end{aligned}
$$

which is similar to eq (5.10).

The harmonic oscillator in an electromagnetic field

The driven reduced initial value problem (6.13) models the motion of a harmonic oscillator in an electromagnetic field, with uniform magnetic field and non-stationary electric field. Indeed, taking $P(q, m)$ a particle with mass $m$ and electric charge $q$, its motion is modeled by the initial value problem:

$$
\left\{\begin{array}{l}
m \ddot{\mathbf{r}}=q(\dot{\mathbf{r}} \times \mathbf{B}+\mathbf{E})-k \mathbf{r} \\
\mathbf{r}(0)=\mathbf{r}_{0} ; \dot{\mathbf{r}}(0)=\mathbf{v}_{0}
\end{array}\right.
$$

where $\mathbf{B}$ is the magnetic field and $\mathbf{E}=\mathbf{E}(t)$ the non-stationary electric field. By denoting:

$$
\boldsymbol{\omega}=\frac{q \mathbf{B}}{2 m} ; \quad \omega_{*}=\sqrt{\frac{k}{m}} ; \quad \frac{q \mathbf{E}}{m}=\mathbf{f},
$$

eq (6.18) becomes the initial value problem (6.13) with the solution given by eq (6.16).

\section{A Tensor Conservation Law of the Foucault Pendulum Problem}

There exists a tensor map that contains all conservation laws of the Foucault Pendulum problem. The notation:

$$
\mathbf{u}^{\otimes 2} \stackrel{\text { def }}{=} \mathbf{u} \otimes \mathbf{u}
$$

is introduced (here " $\otimes "$ represents the diadic product of two vectors). A tensor that will offer all conservation laws of the Foucault Pendulum-like problem is defined. Its eigenvectors are the generalized angular momentum $\boldsymbol{\Omega}=\mathbf{r} \times(\dot{\mathbf{r}}+\boldsymbol{\omega} \times \mathbf{r})$ and the vectorial semiaxes $\mathbf{a}$ and $\mathbf{b}$ of the ellipse from plane $\boldsymbol{\Pi}(t)$. Its eigenvalues are 0 and the squares of the ellipse semiaxes. Its trace will lead to the generalized energy conservation law.

Theorem 7.0.1. The tensor map:

$$
\mathbf{L}=\mathbf{r}^{\otimes 2}+\frac{1}{\omega_{*}^{2}}(\dot{\mathbf{r}}+\boldsymbol{\omega} \times \mathbf{r})^{\otimes 2}
$$


satisfies:

$$
\begin{aligned}
\mathbf{L} \boldsymbol{\Omega} & =\mathbf{0}, \\
\mathbf{L} \mathbf{a} & =a_{0}^{2} \mathbf{a}, \\
\mathbf{L} \mathbf{b} & =b_{0}^{2} \mathbf{b}, \\
\operatorname{trace} \mathbf{L} & =\text { constant }=\frac{2 h}{\omega_{*}^{2}},
\end{aligned}
$$

where $\mathbf{a}=\mathbf{R}_{-\boldsymbol{\omega}} \mathbf{a}_{0}, \mathbf{b}=\mathbf{R}_{-\boldsymbol{\omega}} \mathbf{b}_{0}$ and $\mathbf{a}_{0}, \mathbf{b}_{0}$ are defined in eqs (4.11).

Before proceeding to the proof, remark that the tensor map $\mathbf{L}$ defined in eq (7.2) satisfies:

$$
\mathbf{L}=\mathbf{R}_{-\omega} \mathbf{L}_{0}\left(\mathbf{R}_{-\boldsymbol{\omega}}\right)^{T}
$$

where

$$
\mathbf{L}_{0}=\mathbf{r}_{0}^{\otimes 2}+\frac{1}{\omega_{*}^{2}}\left(\mathbf{v}_{0}+\omega_{0} \times \mathbf{r}_{0}\right)^{\otimes 2}
$$

. Indeed, by computing:

$$
\mathbf{F}_{\omega} \mathbf{L}\left(\mathbf{F}_{\omega}\right)^{T}=\left(\mathbf{F}_{\omega} \mathbf{r}\right)^{\otimes 2}+\frac{1}{\omega_{*}^{2}}\left[\mathbf{F}_{\omega}(\dot{\mathbf{r}}+\boldsymbol{\omega} \times \mathbf{r})\right]^{\otimes 2},
$$

it follows that:

$$
\frac{d}{d t}\left[\mathbf{F}_{\omega} \mathbf{L}\left(\mathbf{F}_{\omega}\right)^{T}\right]=\mathbf{0}_{3},
$$

therefore the tensor map $\mathbf{F}_{\omega} \mathbf{L}\left(\mathbf{F}_{\omega}\right)^{T}$ is constant,

$$
\mathbf{F}_{\omega} \mathbf{L}\left(\mathbf{F}_{\omega}\right)^{T}=\left[\mathbf{F}_{\omega} \mathbf{L}\left(\mathbf{F}_{\omega}\right)^{T}\right]_{t=t_{0}}=\mathbf{L}_{0}
$$

where tensor $\mathbf{L}_{0}$ is defined in eq (7.8). Taking into account that $\left(\mathbf{F}_{\omega}\right)^{T}=\mathbf{R}_{-\boldsymbol{\omega}}$, eq (7.7) holds true.

Proof. Eq (7.6) is proved by taking into account that:

$$
\operatorname{trace} \mathbf{L}=\mathbf{r}^{2}+\frac{1}{\omega_{*}^{2}}(\dot{\mathbf{r}}+\boldsymbol{\omega} \times \mathbf{r})^{2}=\frac{2 h}{\omega_{*}^{2}}
$$

Eq (7.3) is obtained by direct computation.

For eq (7.4) we use that if $\mathbf{u}_{0}$ is eigenvector for $\mathbf{L}_{0}$, then $\mathbf{u}=\mathbf{R}_{-\boldsymbol{\omega}} \mathbf{u}_{0}$ is eigenvector for $\mathbf{L}$. Tensor $\mathbf{L}_{0}$ may be rewritten (see Appendix):

$$
\begin{aligned}
\mathbf{L}_{0} & =\mathbf{r}_{0}^{\otimes 2}+\frac{1}{\omega_{*}^{2}}\left(\mathbf{v}_{0}+\boldsymbol{\omega}_{0} \times \mathbf{r}_{0}\right)^{\otimes 2}=\left(\mathbf{r}_{0}\right)^{\otimes 2}+\left(\mathbf{r}_{0}^{*}\right)^{\otimes 2}= \\
& =\left(\mathbf{a}_{0} \cos \gamma-\mathbf{b}_{0} \sin \gamma\right)^{\otimes 2}+\left(\mathbf{a}_{0} \sin \gamma+\mathbf{b}_{0} \cos \gamma\right)^{\otimes 2}=\mathbf{a}_{0} \otimes \mathbf{a}_{0}+\mathbf{b}_{0} \otimes \mathbf{b}_{0} .
\end{aligned}
$$

It follows that:

$$
\begin{aligned}
\mathbf{L}_{0} \mathbf{a}_{0} & =a_{0}^{2} \mathbf{a}_{0} \\
\mathbf{L a} & =\left[\mathbf{R}_{-\boldsymbol{\omega}} \mathbf{L}_{0}\left(\mathbf{R}_{-\boldsymbol{\omega}}\right)^{T}\right]\left(\mathbf{R}_{-\boldsymbol{\omega}} \mathbf{a}_{0}\right)=\mathbf{R}_{-\boldsymbol{\omega}} \mathbf{L}_{0} \mathbf{a}_{0}=a_{0}^{2} \mathbf{R}_{-\boldsymbol{\omega}} \mathbf{a}_{0}=a_{0}^{2} \mathbf{a} .
\end{aligned}
$$

Eq (7.5) may be proved identically.

The tensor map $\mathbf{L}$ defined in (7.2) offers all the conservation laws of the Foucault Pendulum motion. Its constant trace leads to the energy conservation law, its eigenvectors are the generalized angular momentum and the variable ellipse semiaxes $\mathbf{a}$ and $\mathbf{b}$. Its eigenvalues are 0 (corresponding to the generalized angular momentum) and the squares of the ellipse semiaxes, $a_{0}^{2}$ and $b_{0}^{2}$. The canonical form of the tensor map $\mathbf{L}$ is:

$$
\mathbf{L}=\mathbf{a} \otimes \mathbf{a}+\mathbf{b} \otimes \mathbf{b}=\mathbf{R}_{-\boldsymbol{\omega}}\left[\mathbf{a}_{0} \otimes \mathbf{a}_{0}+\mathbf{b}_{0} \otimes \mathbf{b}_{0}\right]\left(\mathbf{R}_{-\boldsymbol{\omega}}\right)^{T} .
$$

From the expressions of $\mathbf{a}$ and $\mathbf{b}$ another interesting relation between the eigenvectors of the tensor map $\mathbf{L}$ arises:

$$
\omega_{*}(\mathbf{a} \times \mathbf{b})=\Omega .
$$




\section{Conclusions}

The present paper is a comprehensive study of the Foucault Pendulum-like motion problem. The importance of this task is bivalent: to offer a complete closed form vectorial solution to a classic Theoretical Mechanics problem and to offer a general framework for the study of the motion in a central force field with respect to a rotating reference frame. The first task proves to be more necessary since this problem is considered without closed form solution in Theoretical Mechanics textbooks, and an approximate (reduced) model is considered instead. The second task leads to surprising applications in Astrodynamics and Celestial Mechanics, such as the Keplerian motion with respect to a rotating reference frame, tracing the ground-track of a satellite by elementary methods and last but not least the relative Keplerian orbital motion. In fact, from a practical point of view, the latter application appears to be more important, due to the use of spacecraft formations in present and future space missions. Also, terminal rendezvous problems may be treated by using the instruments presented in this paper.

The engineering applications that are presented in this paper are mentioned above: the closed form vectorial expression for the law of motion and the velocity of a satellite with respect to a rotating reference frame that is originated in the attraction center (i.e. in the center of the Earth), the exact expression for the ground-track of a satellite and the closed form vectorial solution to the Keplerian relative dynamics. It is proven that the solution to the problem of the relative orbital motion may be decomposed into a difference among the solutions to two initial value problems, both being Foucault Pendulum-like problems.

The results are presented in a vectorial closed form, so they do not depend on the particular coordinate system that is chosen. The method used to reach these results is purely vectorial, assuring that they apply in any situation that may occur. In some particular situations, including the case of the classic Foucault Pendulum, the results are time-explicit.

In the end, an inedite conservation law for the Foucault Pendulum-like motion is presented. It has rather an aesthetic importance than a pragmatic one, from the point of view of the practical applications. A single entity, that is a symmetric tensor valued function, contains all the conservation laws of the motion.

The approach presented in this paper is structured from general to particular, in order to provide a wider framework, that of the motion under the influence of a central force field with respect to a rotating reference frame, not only the classic problem of the Foucault Pendulum motion.

The reduced Foucault Pendulum problem is also comprehensively studied, since it is the model for the classic Foucault Pendulum that is considered in textbooks. The driven pendulum motion (in the reduced and non-reduced cases) is also studied. Closed form solutions are presented in every situation, with some geometric interpretations and visualizations.

\section{$9 \quad$ Appendix}

In this section, starting from the parametric equation of an ellipse depending on a pair of two conjugate semi-diameters, the vectorial semiaxes of the ellipse and the parametric equation depending on them are determined (see [51]). The degenerated ellipse situation is also treated.

The parametric equation of an ellipse is:

$$
\mathbf{r}(t)=\mathbf{r}_{0} \cos \omega_{*}\left(t-t_{0}\right)+\mathbf{r}_{0}^{*} \sin \omega_{*}\left(t-t_{0}\right),
$$

where $\mathbf{r}_{0}$ and $\mathbf{r}_{0}^{*}$ are conjugate semi-diameters (see [49]) (see Figure 17). Its vectorial semiaxes, denoted $\mathbf{a}_{0}$ and $\mathbf{b}_{0}$, will be computed.

Starting from eq (A1) and from the parametric equation of an ellipse having the vectorial semiaxes $\mathbf{a}_{0}$ and $\mathbf{b}_{0}$ :

$$
\mathbf{r}(t)=\mathbf{a}_{0} \cos \left[\omega_{*}\left(t-t_{0}\right)-\gamma\right]+\mathbf{b}_{0} \sin \left[\omega_{*}\left(t-t_{0}\right)-\gamma\right], \gamma \in[0, \pi),
$$

the vectorial expressions of $\mathbf{a}_{0}$ and $\mathbf{b}_{0}$ will be deduced from:

$$
\mathbf{r}_{0} \cos \omega_{*}\left(t-t_{0}\right)+\mathbf{r}_{0}^{*} \sin \omega_{*}\left(t-t_{0}\right)=\mathbf{a}_{0} \cos \left[\omega_{*}\left(t-t_{0}\right)-\gamma\right]+\mathbf{b}_{0} \sin \left[\omega_{*}\left(t-t_{0}\right)-\gamma\right]
$$

Relation (A3) holds true for any $t \geq t_{0}$. Making $t=t_{0}$ in eq (A3) then $t=t_{0}$ in the derivation of eq (A3), it results:

$$
\mathbf{r}_{0}=\mathbf{a}_{0} \cos \gamma-\mathbf{b}_{0} \sin \gamma, \quad \mathbf{r}_{0}^{*}=\mathbf{a}_{0} \sin \gamma+\mathbf{b}_{0} \cos \gamma .
$$

From eq (A4) it results:

$$
\mathbf{a}_{0}=\mathbf{r}_{0} \cos \gamma+\mathbf{r}_{0}^{*} \sin \gamma, \quad \mathbf{b}_{0}=-\mathbf{r}_{0} \sin \gamma+\mathbf{r}_{0}^{*} \cos \gamma .
$$



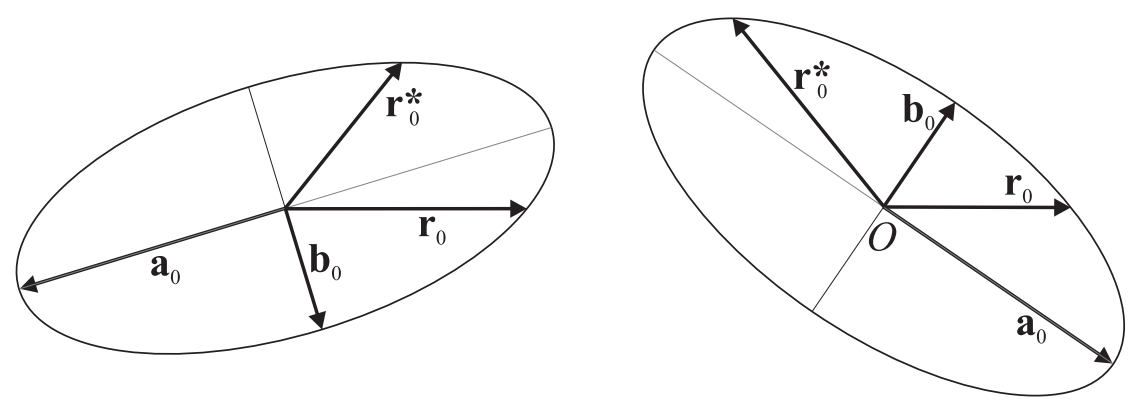

Figure 17: The vectorial semiaxes and the conjugate diameters of an ellipse; Left: $\gamma \in\left(\frac{\pi}{2}, \pi\right)$; Right: $\gamma \in\left[0, \frac{\pi}{2}\right)$.

Since $\mathbf{a}_{0}$ and $\mathbf{b}_{0}$ are the ellipse semiaxes, we impose the conditions:

$$
\begin{aligned}
& \mathbf{a}_{0} \cdot \mathbf{b}_{0}=0 \Rightarrow\left(\mathbf{r}_{0}^{* 2}-\mathbf{r}_{0}^{2}\right) \sin 2 \gamma+2\left(\mathbf{r}_{0} \cdot \mathbf{r}_{0}^{*}\right) \cos 2 \gamma=0 ; \\
& \mathbf{a}_{0}^{2}=\mathbf{r}_{\max }^{2}=\frac{1}{2}\left[\mathbf{r}_{0}^{2}+\mathbf{r}_{0}^{* 2}+\sqrt{\left(\mathbf{r}_{0}^{2}-\mathbf{r}_{0}^{* 2}\right)^{2}+4\left(\mathbf{r}_{0} \cdot \mathbf{r}_{0}^{*}\right)^{2}}\right], \\
& \mathbf{b}_{0}^{2}=\mathbf{r}_{\min }^{2}=\frac{1}{2}\left[\mathbf{r}_{0}^{2}+\mathbf{r}_{0}^{* 2}-\sqrt{\left(\mathbf{r}_{0}^{2}-\mathbf{r}_{0}^{* 2}\right)^{2}+4\left(\mathbf{r}_{0} \cdot \mathbf{r}_{0}^{*}\right)^{2}}\right] .
\end{aligned}
$$

Computing $\mathbf{a}_{0}^{2}$ and $\mathbf{b}_{0}^{2}$ from eq (A5) and after elementary trigonometric computations, it results:

$$
\left\{\begin{array}{l}
\mathbf{a}_{0}^{2}=\frac{1}{2}\left[\mathbf{r}_{0}^{2}+\mathbf{r}_{0}^{* 2}+\left(\mathbf{r}_{0}^{2}-\mathbf{r}_{0}^{* 2}\right) \cos 2 \gamma+2\left(\mathbf{r}_{0} \cdot \mathbf{r}_{0}^{*}\right) \sin 2 \gamma\right] \\
\mathbf{b}_{0}^{2}=\frac{1}{2}\left[\mathbf{r}_{0}^{2}+\mathbf{r}_{0}^{* 2}-\left(\mathbf{r}_{0}^{2}-\mathbf{r}_{0}^{* 2}\right) \cos 2 \gamma-2\left(\mathbf{r}_{0} \cdot \mathbf{r}_{0}^{*}\right) \sin 2 \gamma\right]
\end{array}\right.
$$

From eq (A6) and any of the relations given in eqs (A7) it results that $\gamma \in[0, \pi)$ is uniquely defined by:

$$
\cos 2 \gamma=\frac{\mathbf{r}_{0}^{2}-\mathbf{r}_{0}^{* 2}}{\sqrt{\left(\mathbf{r}_{0}^{2}-\mathbf{r}_{0}^{* 2}\right)^{2}+4\left(\mathbf{r}_{0} \cdot \mathbf{r}_{0}^{*}\right)^{2}}} ; \quad \sin 2 \gamma=\frac{2\left(\mathbf{r}_{0} \cdot \mathbf{r}_{0}^{*}\right)}{\sqrt{\left(\mathbf{r}_{0}^{2}-\mathbf{r}_{0}^{* 2}\right)^{2}+4\left(\mathbf{r}_{0} \cdot \mathbf{r}_{0}^{*}\right)^{2}}}
$$

when $\left(\mathbf{r}_{0}^{2}-\mathbf{r}_{0}^{* 2}\right)^{2}+4\left(\mathbf{r}_{0} \cdot \mathbf{r}_{0}^{*}\right)^{2} \neq 0$. If $\left(\mathbf{r}_{0}^{2}-\mathbf{r}_{0}^{* 2}\right)^{2}+4\left(\mathbf{r}_{0} \cdot \mathbf{r}_{0}^{*}\right)^{2}=0$, it results:

$$
\mathbf{a}_{0}=\mathbf{r}_{0} ; \quad \mathbf{b}_{0}=\mathbf{r}_{0}^{*}
$$

Remark that if $\left(\mathbf{r}_{0}^{2}-\mathbf{r}_{0}^{* 2}\right)^{2}+4\left(\mathbf{r}_{0} \cdot \mathbf{r}_{0}^{*}\right)^{2}=0$, the motion described by eq (A1) is a uniform circular one. The quantity:

$$
c=\sqrt{\left(\mathbf{r}_{0}^{2}-\mathbf{r}_{0}^{* 2}\right)^{2}+4\left(\mathbf{r}_{0} \cdot \mathbf{r}_{0}^{*}\right)^{2}}=\sqrt{\mathbf{a}_{0}^{2}-\mathbf{b}_{0}^{2}}
$$

represents the focal distance of the ellipse that has the parametric equation (A1). The case $c=0$ leads to a circular trajectory. 
When $\mathbf{r}_{0} \times \mathbf{r}_{0}^{*}=\mathbf{0}$, the ellipse degenerates into a line segment. Eq (A1) becomes:

$$
\begin{aligned}
\mathbf{r}(t)= & {\left[r_{0} \cos \omega_{*}\left(t-t_{0}\right)+\frac{\mathbf{r}_{0} \cdot \mathbf{r}_{0}^{*}}{r_{0}} \sin \omega_{*}\left(t-t_{0}\right)\right] \frac{\mathbf{r}_{0}}{r_{0}}=} \\
= & \sqrt{\mathbf{r}_{0}^{2}+\left(\frac{\mathbf{r}_{0} \cdot \mathbf{r}_{0}^{*}}{r_{0}}\right)^{2}} \sin \left[\omega_{*}\left(t-t_{0}\right)+\varphi\right] \frac{\mathbf{r}_{0}}{r_{0}}, \\
\text { where: } & \left\{\begin{array}{l}
\sin \varphi=\frac{r_{0}}{\sqrt{\mathbf{r}_{0}^{2}+\left(\frac{\mathbf{r}_{0} \cdot \mathbf{r}_{0}^{*}}{r_{0}}\right)^{2}}}, \\
\cos \varphi=\frac{\mathbf{r}_{0} \cdot \mathbf{r}_{0}^{*}}{r_{0} \sqrt{\mathbf{r}_{0}^{2}+\left(\frac{\mathbf{r}_{0} \cdot \mathbf{r}_{0}^{*}}{r_{0}}\right)^{2}}},
\end{array}\right.
\end{aligned}
$$

The motion described by (9.1) is a harmonic rectilinear oscillation with pulsation $\omega_{*}$.

When $\mathbf{r}_{0}=\mathbf{0}$ :

$$
\mathbf{r}(t)=\mathbf{r}_{0}^{*} \sin \omega_{*}\left(t-t_{0}\right) .
$$

Eq (A12) describes a harmonic rectilinear oscillation along the direction of vector $\mathbf{r}_{0}^{*}$.

\section{References}

[1] Foucault, M.L., Physical Demonstration of the Rotation of the Earth by Means of the Pendulum, Comptes Rendus de l'Académie des Sciences de Paris, Translated for the Journal of the Franklin Institute (1851), 350-353.

[2] Anonymous, On Foucault's Pendulum Experiments, Journal of the Franklin Institute (1851).

[3] Lancaster, E.R., Relative Motion of Two Particles in Elliptic Orbits, AIAA Journal, Vol.8, No.10 (1970), 1878 1879 .

[4] Bereen, T., Svedt, G., Relative Motion of Particles in Coplanar Elliptic Orbits, Journal of Guidance and Control, Vol. 2 (1979), 443-446.

[5] Humi, M., Fuel-Optimal Rendezvous in a General Central Force Field, Journal of Guidance, Control and Dynamics, Vol. 16, No. 1 (1993), 215-217.

[6] Clohessy, W. H., and Wiltshire, R. S., Terminal Guidance System for Satellite Rendezvous, Journal of the Aerospace Sciences, Vol. 27, No. 9 (1960), 653-658.

[7] Lawden, D. F., Optimal Trajectories for Space Navigation, Butterworth, London (1963).

[8] Tschauner, J., Hempel, P., Optimale Beschleunigeungsprogramme für das Rendezvous-Manover, Acta Astronautica, Vol. 10, (1964), 296-307.

[9] Tschauner, J., The Elliptic Orbit Rendezvous, AIAA 4th Aerospace Sciences Meeting, June 27-29, Los Angeles, CA, (1966).

[10] Gómez, G., and Marcote, M., High-Order Analytical Solutions on Hill's Equations, Celestial Mechanics and Dynamical Astronomy, Vol. 94, No. 2 (2006), 197-211.

[11] Carter, T. E., New Form for the Optimal Rendezvous Equations Near Keplerian Orbit, Journal of Guidance, Control, and Dynamics,Vol. 13, No.1 (1990), 183-186.

[12] Sengupta, P., Dynamics and Control of Satellite Relative Motion in a Central Gravitational Field, Ph.D. Thesis, Texas A\&M University (2006).

[13] Carter, T.E., State Transition Matrices for Terminal Rendezvous Studies: Brief Survey and New Example, Journal of Guidance, Control, and Dynamics, Vol. 21, No. 1 (1998), 148-155. 
[14] Gim, D.W., Alfriend, K.T., The State Transition Matrix of Relative Motion for the Perturbed Non-Circular Reference Orbit, AAS/AAIA Space Flight Mechanics Meeting, San-Antonio, USA (2002).

[15] Balaji, S.K., Tatnall, A., Precise Modeling of Relative Motion for Formation Flying Spacecraft, $54^{\text {th }}$ International Astronautical Congress of the International Astronautical Federation, the International Academy of Astronautics, and the International Institute of Space Law, Bremen, Sep.29 - Oct.3 (2003).

[16] Gurfil, P., Kasdin, N. J., Nonlinear Modeling of Spacecraft Relative Motion in the Configuration Space, Journal of Guidance, Control, and Dynamics, Vol. 27, No. 1 (2004), 154-157.

[17] Baoyin, H., Junfeng, L., Yunfeng, G., Dynamical Behaviors and Relative Trajectories of the Spacecraft Formation Flying, Aerospace Science and Technology, No. 6 (2002), 295-301.

[18] Lee, D., Cochran, J.E., Jo, J.H., Solutions to the Variational Equations for Relative Motion of Satellites, Journal of Guidance, Control, and Dynamics, Vol. 30, No. 3 (2007), 669-678.

[19] Jiang, F., Li, J., Baoyin, Y., Approximate analysis for relative motion of satellite formation flying in elliptical orbits, Celestial Mechanics and Dynamical Astronomy, Vol. 98, No.1 (2007), 31-66.

[20] Gurfil, P., Kholshevnikov, K. V., Distances on the Relative Spacecraft Motion Manifold, AIAA Guidance, Navigation, and Control Conference and Exhibit, San Francisco (2005).

[21] Condurache, D., Martinusi, V., Kepler's Problem in Rotating Reference Frames. Part I: Prime Integrals. Vectorial Regularization, AIAA Journal of Guidance, Control and Dynamics, Vol. 30, No.1 (2007) 192-200.

[22] Condurache, D., Martinusi, V., Kepler's Problem in Rotating Reference Frames. Part II: Relative Orbital Motion, AIAA Journal of Guidance, Control and Dynamics, Vol. 30, No.1 (2007), 201-213.

[23] Condurache, D., Martinusi, V., A Novel Hypercomplex Solution to Kepler's Problem, PADEU, Astron. Dept. of the Eötvös Univ., vol. 19 (2007), 65-80.

[24] Condurache, D., Martinuşi, V., A Closed Form Vectorial Solution to the Relative Orbital Motion, PADEU, Astron. Dept. of the Eötvös Univ., vol. 19 (2007), 49-64.

[25] Condurache, D., Martinusi, V., Relative Spacecraft Motion in a Central Force Field, AIAA Journal of Guidance, Control and Dynamics, Vol. 30, No.3 (2007), 873-876.

[26] Condurache, D., Martinusi, V., Exact Solution to the Relative Orbital Motion in Eccentric Orbits, International Conference "Analytical Methods of Celestial Mechanics", Sankt-Petersburg, Russia, July 8-12 (2007).

[27] Condurache, D., Martinusi, V., A complete closed form vectorial solution to the Kepler problem, Meccanica, Vol. 42, No 5 (2007), 465-476.

[28] Appell, P., Traité de mécanique rationelle (5 vol.), Gauthier-Villars, Paris (1926).

[29] Arnold, V.I., Mathematical Methods of Classical Mechanics, Springer-Verlag, New York (Translated from the 1974 Russian original by K. Vogtmann and A. Weinstein) (1989).

[30] Levi-Civita, T., Amaldi, U., Lezioni di mecanica razionale, Nicola Zanichelli, editore, (1922-1926).

[31] Landau L., Lifschitz E., Mécanique, Éd. Mir, Moscou (1981).

[32] Goldstein, H., Poole, C.P., Safko, J.L., Classical Mechanics, 3rd ed., Addison Wesley (2002).

[33] Lebedev, L.P., Cloud, M.J., Tensor Analysis, World Scientific Publishing (2003).

[34] Geradin, M., Cardona, A., Flexible Multibody Dynamics - A Finite Element Approach, John Wiley and Sons (2001).

[35] Simmonds, J.G., A Brief on Tensor Analysis, 2nd ed., Springer (1994).

[36] Teodorescu, P.P., Mechanical Systems, Classical Models. Volume 1: Particle Mechanics, Springer (2007). 
[37] Inalhan, G., How, J.P., Relative Dynamics \& Control of Spacecraft Formations in Eccentric Orbits, AIAA Guidance, Navigation, and Control Conference and Exhibit, Denver, CO (2000).

[38] Inalhan, G., Tillerson, M., How, P., Relative Dynamics and Control of Spacecraft Formations in Eccentric Orbits, Journal of Guidance, Control and Dynamics, Vol. 25, No. 1 (2002).

[39] Zhang, H., Sun, L., Spacecraft Formation Flying in Eccentric Orbits, AIAA Guidance, Navigation and Control Conference and Exhibit, Austin, Texas (2003).

[40] Breger, L.S., Model Predictive Control for Formation Flying Spacecraft, submitted to the Department of Aeronautics and Astronautics in partial fulfillment of the requirements for the degree of Master of Science in Aeronautics and Astronautics, MIT (2004).

[41] Sengupta, P., Satellite Relative Motion Propagation and Control in the Presence of $J_{2}$ Perturbations, Submitted to Texas A\&M University in partial fulfilment of the requirements for the degree of Master of Science (2003).

[42] Darboux, G., Leçons sur la théorie générale des surfaces et les applications géométriques du calcul infinitesimal, Tome 1, Chap. 2, Gautiers-Villars, Paris (1887).

[43] Lurie, A.I, Analytical Mechanics, Springer (2002).

[44] Fasano, A., Marmi, S., Analytical Mechanics, Oxford University Press (2006).

[45] Marsden. J.E., Ratiu, T.S., Introduction to Mechanics and Symmetry, Springer (1994).

[46] Angeles, J., Fundamentals of Robotic Mechanical Systems: Theory, Methods, and Algorithms, Springer (2006).

[47] Angeles, J., Rational Kinematics, Springer (1989).

[48] Gantmacher, F.R., The Theory of Matrices, New York, Chelsea (1959).

[49] Comberousse, R.E., Traité de géométrie (deuxième partie), Gauthier-Villars, Paris (1922).

[50] Condurache, D., New Symbolic Methods into the Study of Dynamic Systems (in Romanian), Ph.D. thesis, Technical University "Gh Asachi" Iaşi (1995).

[51] Condurache, D., Symbolic Representations. Application in Signal Theory and Dynamical Systems Study (in Romanian), Nord-Est, (1996).

[52] Condurache, D., Matcovschi, M.H., An Exact Solution to Foucault's Pendulum Problem, Bul. Inst. Polit. Iasi, Vol. XLI (XLVII), Fasc. 3-4, section Mathematics, Theoretical Mechanics, Physics (1997), 83-92..

[53] Condurache, D., Matcovschi, M.H., A General Method to Obtain an Exact Vectorial Solution to Foucault's Pendulum Problem, Bul.Inst.Polit.Iasi, Vol. XLVI(L), Fasc 1-2, section Mathematics, Theoretical Mechanics, Physics (2000), 79-96.

[54] Battin, R.H., An Introduction to the Mathematics and Methods of Astrodynamics, AIAA (1999).

[55] Madonna, R.G., Orbital Mechanics, Krieger Publishing Company, Malabar, Florida (1997).

[56] Roy, A.E., Orbital Motion, Institute of Physics Publishing, Bristol and Philadelphia (2005).

[57] Hill, G.W., Researches in Lunar Theory, American Journal of Mathematics, Vol. 1 (1878), 5-26. 